\title{
Nonlinear Dynamics of Magnetic Bearing Systems
}

This is a literature review paper and published in

International Journal of Material Systems and Structures

Vol. 19, Issue 12, 2008, pp.1471-1491.

J.C. Ji, Colin H. Hansen, Anthony C. Zander

School of Mechanical Engineering

The University of Adelaide

SA 5005, AUSTRALIA

Current email address: jin.ji@uts.edu.au

Abstract

Magnetic bearings use magnetic forces to support various machine components. Because of the non-contact nature of this type of suspension, magnetic bearing technology offers a number of significant advantages over conventional bearings such as rolling element and fluid film bearings. An active magnetic bearing basically consists of an electromagnetic actuator, position sensors, power amplifiers, and a feedback controller. All of these components are characterized by nonlinear behaviour and therefore the entire system is inherently nonlinear. However, in simulations of the dynamic behaviour of magnetic bearing systems, the nonlinearities are usually neglected to simplify the analysis and only linear models are used. Moreover, many control techniques currently used in magnetic bearing systems are generally designed by ignoring nonlinear effects. The main reason for simplification is the intractability of the complexity of the actual model. In fact, the inherent nonlinear properties of magnetic bearing systems can lead to dynamic behaviour of a magnetically suspended rotor that is distinctly different from that predicted using a simple linearized model. Therefore the nonlinearities should be taken into account. 
This literature review is focussed on the nonlinear dynamics of magnetic bearing systems and it provides background information on analytical methods, nonlinear vibrations resulting from a rotor contacting auxiliary bearings, and other active topics of research involving the nonlinear properties of magnetic bearing systems such as nonlinear selfsensing magnetic bearings and nonlinear control of magnetic bearings. The paper concludes with a brief discussion on current and possible future directions for research on the nonlinear dynamics of magnetic bearing systems.

Keywords: magnetic bearing systems, nonlinear vibrations, stability, bifurcations, periodic motion, chaotic motion, time delays, nonlinear rotor-dynamics, nonlinear dynamic behaviour. 


\section{Introduction}

One of the most innovative developments in the turbomachinery field involves the use of active magnetic bearings for rotor support. Magnetic bearings use magnetic forces to support moving machinery without physical contact. Because of the non-contact nature of the suspension, this new bearing technology offers a number of significant advantages over conventional bearings such as rolling element and fluid film bearings. These advantages include elimination of the lubrication system, very low friction, no wear, high rotor speed, and adjustable dynamic properties. Magnetic bearings can offer a high loadcarrying capability by optimising system and material parameters including the bearing air gap, bearing material saturation flux, bearing surface area, number of bearing coil turns and amplifier power. Magnetic bearings can permit operation in extreme environments such as high temperatures, low temperatures and vacuums. An advanced monitoring system incorporated in a magnetic bearing system can not only monitor instantaneous system parameters such as rotor position, lateral and axial vibration, electrical current, temperature and rotational speed, but can also analyse the unbalance by calculating its location and magnitude. The electronic controllers can change bearing stiffness and damping properties, allowing for adjustments to system dynamics that affect resonance frequencies and reduce transmitted vibration. Magnetic bearings integrated into a rotorbearing system may be used for synchronous disturbance control and vibration control [Knospe, Hope, Fedigan and Williams, 1995; Matsushita, Imashima and Okubo, 2002; Cole, Keogh and Burrows, 2002; Kasarda, Mendoza, Kirk and Wicks, 2004; Shi, Zmood and Qin, 2004]; vibration suppression and attenuation [Knospe and Tamer, 1997; Cole, Keogh and Burrows, 1998; Keogh, Cole and Burrows, 2002; Johnson, Nascimento, Kasarda and Fuller, 2003]; for active health monitoring of rotordynamic systems [Mani, 
Quinn and Kasarda, 2006]; and on-line identification and fault diagnosis [Aenis, Knopf and Nordmann, 2002; Quinn, Mani, Kasarda, Bash, Inman and Kirk, 2005].

The application of magnetic bearing technology has experienced substantial growth during the past two decades, since the First International Symposium on Magnetic Bearings was held in 1988 [Schweitzer, 1988]. Meanwhile, considerable research has been undertaken to cover all aspects of magnetic bearings including sensing and control technology, modelling and identification, components and materials, and self-sensing techniques [Higuchi, 1990; Allaire, 1992; Schweitzer, Siegwart and Herzog, 1994; Matsumura, Okada, Fujita and Namerikawa, 1996; Allaire and Trumper, 1998; Schweitzer, Siegwart, Loesch and Berksun, 2000; Okada and Nonami, 2002; Lyndon and Trumper, 2004; Bleuler and Genta, 2006]. Significant progress has been made in understanding key issues in designing reliable magnetic bearings. Magnetic bearings have now moved beyond promise into actual service in such applications as turbomachinery, centrifuges, vacuum machinery, machine tool spindles, medical devices, robotics, high-speed drives, spacecraft equipment, contactless actuators and vibration isolation [Kasarda, 2000]. Magnetic bearings are also used in high-precision instruments and to support equipment in a vacuum, for example in flywheel energy storage systems. A flywheel in a vacuum has very low windage losses, but conventional bearings usually fail quickly in a vacuum due to poor lubrication. This literature review will summarize the development of current research in understanding the nonlinear dynamics of magnetic bearings, with a focus on the effects of nonlinear properties and time delays on the nonlinear dynamics and dynamic stability of magnetic bearing systems.

This section is organized into three subsections. Subsection 1.1 presents a brief introduction to magnetic bearings and active topics of current research relevant to 
magnetic bearing technology. Section 1.2 discusses the nonlinear properties of magnetic bearings and gives reasons for the need to consider the nonlinear dynamic analysis of magnetic bearing systems. Section 1.3 outlines the organization of this literature review.

\subsection{Magnetic bearings and the active topics of research}

A bearing is a component used to reduce friction in a machine. Bearings may be classified broadly as radial bearings and thrust bearings according to the motions they allow. Alternatively, bearings may be grouped according to six common principles of operation: namely sliding bearings; rolling element bearings; fluid bearings; flexure bearings and magnetic bearings.

\section{[Insert Figure 1 here ]}

Magnetic bearings use magnetic forces to support moving machinery without physical contact. The stable operation of a magnetic bearing system can only be achieved by feedback control. Conceptually, a typical active magnetic bearing is composed of four basic components; position sensors, feedback controllers, power amplifiers, and electromagnetic actuators. Figure 1 shows a block diagram of a magnetic bearing system. The non-contact position sensor is used to measure the position of the shaft, and this signal is used by the controller to generate the control signals, which are fed into the power amplifier, which in turn supplies the required currents to each of the actuator coils. Finally, the electromagnets generate the suspension and operating forces.

Figure 2 shows a single-degree-of-freedom magnetic system with a pair of opposed electromagnets in combination (commonly referred to as a two-pole magnetic bearing) to 
provide magnetic attractive forces, where $I_{1}$ and $I_{2}$ represent the currents flowing in the coils, $g_{0}$ denotes the nominal air gap between the rotor and electromagnets, and $x$ designates the displacement of the geometrical centre of the rotor from the centre of the magnetic bearing. This simple model, without unnecessary complexity, represents a fundamental structure for many more complicated magnetic bearing arrangements. Using this model, many researchers have designed control systems and self-sensing magnetic bearings as well as examined the stability and dynamics of simple rotor-bearing systems.

\section{[Insert Figure 2 here ]}

It should be mentioned that a rotating machine with active magnetic bearings is commonly equipped with conventional bearings as a backup support system in the event of failure of the magnetic bearings. The backup support system is usually referred to as auxiliary bearings or backup bearings in the literature. The auxiliary bearing system carries no load during normal system operation and is designed to provide machine protection in the unlikely event of an electronic failure or power failure, which would cause loss of magnetic support and subsequent rotor delevitation. The loss of the magnetic bearing function during operation may lead to either a transient or persistent contact event between the auxiliary bearings and the magnetically suspended rotor. Subsequent interactions of the rotor and auxiliary bearings may significantly influence the behaviour of the rotor through producing very large amplitude vibrations and high instantaneous loads. A deep understanding of the dynamics of the rotor contacting auxiliary bearings is essential to help design better auxiliary bearings.

Research relevant to magnetic bearings has received considerable interest from research groups and industry engineers since the First International Symposium on Magnetic 
Bearing Systems was held in Zurich, Switzerland in 1988 [Schweitzer, 1988]. The literature on magnetic bearings is huge and diverse, primarily due to a wide variety of both theoretical research and practical applications. Hundreds of papers appear every year in academic journals, conference proceedings and technical reports. The currently active topics of research on magnetic bearings as indicated by the topics of interest for past international symposiums on magnetic bearings can be classified by specific subjects covering all the aspects of research and applications including: active magnetic bearings; passive magnetic bearings; superconductor magnetic bearings; micro bearings; magnetic actuators; new sensing and control technology; industrialization, safety and reliability aspects; modelling and identification; field experiences and case studies; components and materials; self-bearing motors; self-sensing techniques; and application of magnetic bearings for vibration control and online diagnosis.

Active magnetic bearings may be currently perceived as reaching a mature state. Their applications are steadily increasing in number while new application fields are emerging. It is expected that new fields of research will continue to appear to keep track with increasing numbers of applications. This paper presents a literature review of nonlinear dynamics and dynamic stability of magnetic bearing systems.

\subsection{Nonlinear properties of magnetic bearing systems}

As shown in Figure 1, a magnetic bearing system is basically composed of sensors, controllers, amplifiers and electromagnets. All of these components are characterized by nonlinear behaviour and therefore the entire system is inherently nonlinear. The most important nonlinearities are: 
1). The nonlinear magnetic force to displacement and force to coil current relationships (or nonlinear force to magnetic flux relationship) of the electromagnets;

2). The geometric coupling between the electromagnets, which results in coupling between different orthogonal coordinate directions;

3). The saturation of the ferromagnetic core material, which results in a flattening of the magnetization curve;

4). The hysteresis of the magnetic core material;

5). The saturation of the power amplifier and the limitation of the control current, which are caused by physical limitations of the power amplifier;

6). The unavoidable time delays in the controller and actuators, especially when the control algorithm is implemented on a digital signal processor;

7). The nonlinearity and noise of the sensor system;

8). The nonlinearity of the coil inductance; and

9). The eddy current effect, the leakage and fringing effect, and the nonlinear magnetization B-H curve.

When linear modelling is used to characterise magnetic bearings, the nonlinear electromagnetic forces are linearized about the operating point and considered to be a linear function of currents and air-gaps. The linearized magnetic forces may alternatively be expressed in terms of spring stiffness and damping, such as given by Tonoli and Bornemann [1998]; Kim and Lee [1999]; Peel, Bringham and Howe [2000]. However, the linear relationship holds only locally and the linear behaviour of rotor motion can be approximated only for small rotor deflections and small control currents. If the rotor deflections exceed half the gap, the net magnetic force of an opposite pair of electromagnets differs by more than $44 \%$ from its linear approximation [Skricka and Markert, 2002]. Consequently, the performance of magnetic bearings may suffer rapid 
deterioration when the operation deviates from the equilibrium point. In practice, the nonlinear characteristics become quite significant for large control currents and small airgaps. The nonlinear properties of magnetic bearings can lead to dynamic behaviour of rotor motion that is totally different from that predicted by a linear model. A nonlinear dynamic analysis of rotor-magnetic bearing systems is required in order to fully utilize the capacity of a magnetic bearing. Investigation of the effects of the nonlinearities on the stability and dynamics as well as on the performance of magnetic bearings has received significant attention of the international research community in the past decade.

There is a strong need for the dynamic analysis of magnetic bearing systems to be nonlinear, at least for two main reasons. First, a fundamental scientific investigation of the effect of nonlinearities on the dynamic behaviour of magnetic bearings can provide valuable insights into system characteristics under various operating conditions, and predict the complicated dynamic behaviour of the system. Second, a precise parametric model of magnetic bearings is required for the optimal design to achieve reliable and stable operation to be realised. The success of any magnetic bearing is highly dependent on the design of the controller that is used to control it. In turn, the controller relies heavily on a priori knowledge of the system dynamics. If the system model is not precisely known, the controller, which is designed for a specific purpose or aimed at compensation of a specific component of the nonlinearities, may fail to meet the performance requirements for the practical system.

As will be discussed in sections 3, 4 and 6, the primary objective of existing studies on the nonlinear dynamics of magnetic bearing systems has been to gain a deeper insight into the effects of the inherent nonlinearities of magnetic bearings and the influence of unavoidable time delays occurring in the feedback control path on the stability, dynamic 
behaviour and performance of magnetic bearing systems. Emphasis has been on stability analysis and local bifurcation control as well as on all aspects of nonlinear dynamic behaviour including bifurcations, co-existence of multiple solutions and amplitudemodulated motions.

\subsection{Organization of this literature review}

Since the First International Symposium on Magnetic Bearings was held in 1988 [Schweitzer, 1988], considerable research has been conducted to study all the aspects of magnetic bearings and their potential applications. This review will not cover all the aspects of research and applications relevant to magnetic bearings, such as new developments in sensing and control system technology and new magnetic bearing system designs. This review will focus on summarising recent research on the nonlinear dynamics of magnetic bearings. Emphasis is given to the nonlinear dynamic behaviour and stability of a rotor supported by magnetic bearings in the presence of the single or multiple nonlinear components which inherently exist in magnetic bearing systems.

The writing of this literature review has proved to be a difficult task in part because the literature on magnetic bearings is growing rapidly every year and across a large number of international journals and conference proceedings, and because the subject has an interdisciplinary nature covering mechanical engineering, electrical engineering and applied mathematics. Even in the context of the nonlinear dynamics of magnetic bearing systems, classification of published studies is a formidable task, as this classification is inevitably biased toward the areas with which the authors are most familiar and have conducted research in. Research topics addressed in the present review are categorized into five main groups: nonlinear vibrations of a rotor contacting auxiliary bearings; 
nonlinear dynamics of one-degree-of-freedom (one-DOF) nonlinear rotor-bearing systems; nonlinear dynamics of two-DOF nonlinear rotor-bearing systems; stability and dynamics of rotor-bearing systems with time delays; and other issues relevant to nonlinear magnetic bearing systems. Note that all the papers cited in this review were written in English. Some non-English papers have been omitted because of their unavailability.

The remaining parts of this literature review are organized as follows: Section 2 briefly describes the analytical methods that have been used in analysis of the nonlinear dynamics of magnetic bearing systems. Section 3 reviews the nonlinear dynamics of simple rotormagnetic bearing systems for which the equations of motion are mathematically modelled by one-DOF nonlinear systems. Section 4 reviews the nonlinear dynamics of magnetic bearing systems whose mathematical modelling is governed by a set of two-DOF nonlinear systems. Section 5 briefly reviews the nonlinear vibrations of a rotor contacting backup auxiliary bearings. The effect of time delays on the stability and dynamics of rotor-magnetic bearing systems is discussed in Section 6. Section 7 presents two emerging topics of research dealing with the nonlinear properties of magnetic bearings: nonlinear self-sensing magnetic bearings; and nonlinear control of magnetic bearings. Section 8 concludes the review by briefly summarizing recent research and development on the nonlinear dynamics of magnetic bearing systems, and by discussing possible topics of future research in the area.

\section{Analytical methods}

Analysis of dynamic behaviour has always been an important aspect in the design and assessment of rotor-bearing systems. Nonlinear rotor motion in rotating machinery is commonly caused by the nonlinear characteristics of the supporting bearings. The 
bearings could be either conventional mechanical bearings (such as ball, journal, or fluidfilm bearings) or magnetic bearings. In general, the forced response of rotor-bearing systems may exhibit periodic, sub-harmonic, super-harmonic and chaotic behaviour as well as saddle node and Hopf bifurcations. Here bifurcations (i.e., local bifurcations) refer to qualitative changes in the structure of the solutions of a system when certain system parameters are varied [Guckenheimer and Holmes, 1983]. Local bifurcations of the forced response of rotor-bearing systems can be analysed entirely through changes in the local stability properties of equilibria or periodic solutions. The stability of steady state solutions can be examined by computing the eigenvalues of the coefficients matrix of the characteristic equations, which are derived from the averaged equations in terms of small disturbances to the steady state solutions. A saddle-node bifurcation corresponds to the real part of an eigenvalue passing through zero. A pitchfork bifurcation associates with a real eigenvalue crossing the imaginary axis into the right-half of the complex plane along the real axis. A Hopf bifurcation is defined as the change in qualitative behaviour when a pair of complex conjugate eigenvalues passes through the imaginary axis. Saddle-node bifurcations usually lead to the phenomenon of bistability, where in a certain interval of the control parameter, two stable attractors exist with an unstable one in-between. Bistability is responsible for hysteresis and jump phenomena. A co-existence of two stable motions may be possible after a pitchfork bifurcation occurs. For a rotor-magnetic bearing system without external excitations, the trivial equilibrium may lose its stability through a Hopf bifurcation and bifurcate into a periodic solution [Ji, 2003; Ji and Hansen, 2005]. Through Hopf bifurcations, the steady state response of an unbalanced rotor may result in amplitude- and phase-modulated motions [Ji and Hansen, 2001; Ji and Leung, 2003]. 
For a rotor supported by rolling bearings, the nonlinear behaviour of the resultant rotorbearing system results mainly from nonlinear Hertzian contact force, bearing clearances, and surface waviness [for example, Pavlovskaia, Karpenko and Wiercigroch, 2004; Harsha, 2006]. For a rotor supported by fluid film bearings, nonlinear hydrodynamic forces are a primary source of vibration and introduce the nonlinear dynamic behaviour of rotor motion [Ding, Cooper and Leung, 2002; Cveticanin, 2005].

For a rotor suspended by magnetic bearings, the nonlinear oscillations of rotor motion may result from either accidental contacts between the rotor and auxiliary bearings or the inherent nonlinearities of magnetic bearing systems. In the former case, the clearance between the rotor and the inner race of the auxiliary bearing introduces a nonlinear dynamic feature after the magnetic bearings fail. As discussed in Section 1.2, the inherent nonlinear properties of magnetic bearing systems are different from those of conventional bearings in terms of types and characteristics, partially because the nonlinear magnetic forces are dependent on control currents or voltages (i.e. magnetic flux).

In comparison with the research on the nonlinear dynamic behaviour of a rotor supported by conventional mechanical bearings (which are either rolling element bearings or fluidfilm bearings), research on the nonlinear dynamic behaviour of a rotor supported by magnetic bearings is far from intensive, mainly because the application of this new bearing technology is less extensive than the application of conventional mechanical bearings, although the use of magnetic bearings for turbomachinery has experienced substantial growth since the First International Symposium on Magnetic Bearings was held in 1988 [Schweitzer, 1988]. 
Fortunately, the growing engineering requirements for the nonlinear analysis of the dynamic behaviour of magnetic bearing systems have been paralleled by a notable advance in dynamical systems theory [Guckenheimer and Holmes, 1983; Wiggins, 1990], which permitted the exploration of several typical nonlinear phenomena. Phrases like nonlinear resonances, bifurcations and chaos are now well documented and understood.

In modelling rotor-magnetic bearing systems in the presence of nonlinearity, the equations of motion governing the response of a magnetically suspended rotor are usually characterised by a set of either one- or two-DOF nonlinear differential equations with quadratic and cubic terms. The closed form of the solutions to these nonlinear differential equations cannot be found analytically, therefore either numerical integration solutions or approximate solutions obtained using a perturbation method have been sought to investigate the nonlinear response of magnetic bearing systems.

In solving nonlinear differential equations, numerical integration schemes such as the Runge-Kutta algorithm are commonly used to find periodic, quasi-periodic and chaotic solutions, and numerical methods such as the continuation method [e.g., Parker and Chua, 1989] may be used to obtain unstable solutions.

There are currently many asymptotic perturbation methods available for finding approximate periodic solutions for nonlinear systems. These perturbation techniques include the averaging method [Hale, 1971], the method of multiple scales [Nayfeh and Mook, 1979], the harmonic balance method [Kim and Choi, 1997], the trigonometric collocation method [Chinta and Palazzolo, 1998], and an asymptotic perturbation method incorporating the harmonic balance method and the method of multiple scales [Maccari, 1998]. A perturbation method is employed to obtain a set of two or four averaged 
equations that determine the amplitudes and phases of the forced response of the rotor motion under primary resonances or secondary resonances. Floquet theory [Hayashi, 1964; Rudiger, 1994] is utilized to study the local stability of periodic solutions. Usually, a perturbation analysis is carried out up to the first-order approximation if the nonlinear systems involve cubic nonlinear terms only, since the higher-order approximate terms do not influence the qualitative behaviour of the asymptotic solutions. On the other hand, if the nonlinear systems involve both quadratic and cubic nonlinearities, second-order approximate solutions are sought, because the quadratic nonlinearities cannot appear in the first-order approximate solutions.

In studying the effect of time delays on the dynamics and stability of rotor motion, the dynamic response of the rotor is mathematically modelled by either one- or two-DOF nonlinear differential equations with time delays. Such systems are usually referred to as functional differential equations in the context of mathematics [Halanay, 1966; Hale, 1977; Hale and Verduyn Lunel, 1983]. The decomposition theory and centre manifold theorem [Halanay, 1966; Hale, 1977; Hale and Verduyn Lunel, 1983; Troger and Steindl, 1991] are used to perform a reduction of an infinite dimensional nonlinear system to a set of two- or four-dimensional ordinary differential equations. A perturbation method is then used to explore the bifurcating solutions and forced response of the system in the neighbourhood of Hopf bifurcations.

\section{One-DOF nonlinear rotor-bearing systems}

Active magnetic bearings use magnetic forces to support various machine components [Schwitzer, Bleuler and Traxler, 1994]. An active magnetic bearing consists of an electromagnetic actuator, position sensors, power amplifiers, and a feedback controller. 
Each actuator is composed of a ferromagnetic component attached to the rotor and its counterpart of stationary electromagnets (known as the stator). In early simulations of the dynamic behaviour of magnetic bearing systems, nonlinearities were usually neglected for simplicity and the components of magnetic bearing systems were simplified to linear models. However, the nonlinear properties of magnetic bearings can lead to behaviour of the rotor-bearing system that is distinctly different from that predicted using a simple linear model. It has been shown that the standard linear magnetic bearing model which is obtained from linearization based on the bias current was imprecise for the control axes affected by gravity, and that special attention was paid to account for the nonlinear effects in the case of non-canonical choice of bias current [Loesch, 2001].

At an early stage of analysis, a two-pole, single-degree-of-freedom (single-DOF) magnetic bearing system was used to study the nonlinear dynamics and stability of the rotor motion. This simple model, as shown in Figure 2 of Section 1.1, without unnecessary complexity, represents a fundamental structure for the analysis of many more complicated magnetic bearings. The equation of motion for a rigid rotor has the form

$$
m \ddot{x}+F_{x}=m e \omega^{2} \cos (\omega t),
$$

where $x$ is the displacement, $m$ is the mass of the rotor, $F_{x}$ is the total magnetic force acting on the rotor in $x$ direction, $e$ is the mass imbalance eccentricity of the rotor, $\omega$ is the rotational speed of the rotor, and a overdot denotes the differentiation with respect to time $t$. The magnetic force can be written as $F_{x}=k\left[I_{2}^{2} /\left(g_{0}-x\right)^{2}-I_{1}^{2} /\left(g_{0}+x\right)^{2}\right]$, where $I_{1}$ and $I_{2}$ are the currents flowing through the coils, $g_{0}$ is the nominal air gap between the stator and the shaft when $x=0$, and $k$ is a constant consisting of the parameters of the electromagnets. The equations of motion governing the dynamics of a magnetically suspended rotor by a two-pole magnetic bearing are mathematically modelled by one- 
DOF nonlinear systems that are expressed in a similar form to equation (1). There are a number of studies in the literature that partially dealt with the problem of the nonlinear modelling and nonlinear dynamics of magnetic bearing systems by using one-DOF nonlinear systems and they are discussed in the following paragraphs.

Mohamed and Emad [1993] used a numerical method to analyse the nonlinear oscillation of a rigid rotor in two radial active magnetic bearings. It was shown that the system undergoes Hopf bifurcation due to unstable periodic motion. However, only a nonlinear force to magnetic flux relationship was considered along with the rotor gyroscopic effects, while other nonlinearities such as geometric coupling, hysteresis and saturation of the magnetic material, and time delays of the feedback controller were neglected. Laier and Markert [1995] carried out a numerical simulation of the nonlinear effects on magnetically suspended rotors. Jump phenomena were found to occur in the system, as shown in Figure 3 , where the frequency-response curves show typical jumps from one stable branch to the other during running up or running down.

\section{[Insert Figure 3 here ]}

Springer, Schlager and Platter [1998] developed a nonlinear model including nonlinear magnetic force and magnetic saturation for radial magnetic bearing actuators. The transient vibration was analysed using a numerical integration procedure. However, they did not consider geometric coupling, time delays occurring in the control system and limitations of the power amplifier and control current. Steinschaden and Springer [1999a] developed a simple nonlinear model containing only the nonlinear force to displacement and force to coil current relationship to investigate the dynamic behaviour of a radial active magnetic bearing system. It was shown that their simple model could exhibit 
symmetry breaking and period doubling bifurcations. However, the other important components of nonlinearity such as geometric coupling, hysteresis, saturation of the magnetic material, and time delays of the control system were neglected. Later, Steinschaden and Springer [1999b] studied the effects of saturation of the proportionalintegral-derivative (PID) controller output and nonlinear magnetic force on the dynamic characteristics of a single-mass rotor by using numerical simulation. It was found that symmetry breaking and quasi-periodic solutions might take place for specific parameter sets. However, they did not take the geometric coupling and time delays of the control system into account. Ji, Yu and Leung [2000] studied the bifurcation of rotor motion in the horizontal and vertical directions near the degenerate point of a double-zero eigenvalue by using the normal form method. The nonlinear magnetic force was expanded about the equilibrium point into a Taylor series of up to the third-order. It was shown that the vibratory behaviour in the vertical direction could be reduced on the centre manifold to the Bogdanov-Takens form [Guckenheimer and Holmes, 1983]. Saddle-node, Hopf and saddle-connection bifurcations were found in the reduced normal form equations. However, other nonlinearities such as geometric coupling, saturation of the power amplifier, hysteresis and saturation of the magnetic material, and time delays of the control system were neglected.

Ji [2004] developed a periodically forced single-DOF piecewise linear system model subjected to a saturation constraint to study the dynamics of a rotor supported by a twopole magnetic bearing with proportional feedback control, in which the actuator is subject to saturation constraints. The magnetic force was simplified to be of a piecewise linear characteristic and the equations of motion in the non-dimensional form is given by

$$
\ddot{y}+2 \delta \ddot{y}+y+\omega_{2}^{2} y=f \cos (\omega t) \quad \text { for }|y| \leq 1,
$$




$$
\ddot{y}+2 \delta \ddot{y}+y+\omega_{2}^{2} \operatorname{sgn}(y)=f \cos (\omega t) \quad \text { for }|y| \geq 1
$$

where $y$ is the dimensionless displacement, $\delta$ is the damping coefficient, and $\operatorname{sgn}(\cdot)$ denotes the sign function.

\section{[Insert Figure 4 here ]}

A symmetric periodic solution with a double-entering saturation region per cycle, as shown in Figure 4, was analytically constructed to represent the motion of a rotor entering the saturation regime twice per cycle of the external force. The periodic solution consists of four distinct segments of the motion according to four time intervals; $\left[t_{0}, t_{1}\right],\left[t_{1}, t_{2}\right]$, $\left[t_{2}, t_{3}\right]$ and $\left[t_{3}, t_{4}\right]$, where $t_{4}=t_{0}+T, T$ is the period of the periodic motion and $t_{i}$ denotes the time instant that the motion crosses the boundaries of the saturation regions $y= \pm 1$. Other kinds of solutions such as asymmetric, subharmonic and chaotic solutions as well as solutions involving a multiple-crossing saturation region per cycle periodic solutions, were found through numerical simulations to exist in the forced response of the system. Ji and Hansen [2004a] constructed an analytical approximate solution for the primary resonance response of a periodically excited piecewise nonlinear-linear oscillator which results from the dynamic modelling of a rotor supported by a magnetic bearing subjected to saturation constraints. The magnetic force of a two-pole magnetic bearing subjected to saturation constraints was modelled to be of linear-nonlinear characteristic. Without eliminating the secular terms, a valid asymptotic expansion solution for the weakly nonlinear equation was analytically determined for the case of primary resonances. A symmetric periodic solution for the overall system was then obtained by imposing continuity and matching conditions. The stability characteristic of the symmetric periodic solution was examined by investigating the asymptotic behaviour of perturbations to the steady state solution. 
Later, Ji and Hansen [2004b] analytically constructed a global symmetric period-1 approximate solution for the non-resonant periodic response of the nonlinear-linear oscillator. The period-1 solution was referred to as the large amplitude motion entering saturation regions twice per period of the external excitation. The approximate solutions were found to be in good agreement with the exact solutions that were obtained from the numerical integration of the original equations. In addition, the dynamic behaviour of the oscillator was numerically investigated with the help of bifurcation diagrams, Lyapunov exponents, Poincare maps, phase portraits and basins of attraction. The existence of subharmonic and chaotic motions and the coexistence of four attractors were observed for some combinations of the system parameters.

\section{[Insert Figure 5 here ]}

Figures 5(a) and (b) show a bifurcation diagram and the maximum Lyapunov exponent (mLe) with an increase of the forcing frequency $\Omega$ in the region $\Omega \in[0.37,0.49]$, where $\Omega$ is defined as the forcing frequency. The Lyapunov exponents were calculated using the algorithm derived by Wolf, Swift, Swinney and Vastano [1985] and iterated over at least 1000 forcing periods. There exist period-1, period-2 and period-4 motions as well as chaotic motions in the given interval of forcing frequency. At $\Omega \cong 0.4025$, the maximum Lyapunov exponent changes from a negative to a positive value. For $0.4025<\Omega<0.466$, the maximum Lyapunov exponent is positive, thereby confirming the occurrence of chaotic motions. At $\Omega \cong 0.3975,0.4025$, and 0.483 , the maximum Lyapunov exponent is nearly zero, which corresponds to the occurrence of a bifurcation. A sequence of period doubling bifurcations leading to chaotic motion was observed in Figure 5(a) in the range $\Omega \in[0.37,0.44]$, which is a typical route to chaos observed in a large range of mechanical 
systems. The chaotic motion disappears through reverse period doubling bifurcations, firstly bifurcating to a period-4 then to a period-2, and eventually settling to a period-1 motion.

\section{[Insert Figure 6 here ]}

Figure 6 shows the phase portrait and Poincare map of a chaotic motion of the system for $\Omega=0.45$. The two Lyapunov exponents for the chaotic motion are approximately 0.139363 and 0.386685, respectively. Later, Ji and Hansen [2005a] applied a matching method and a modified averaging method to construct an approximate solution for the super-harmonic resonance response of the periodically excited nonlinear oscillator with a piecewise nonlinear-linear characteristic. The validity of the developed analysis was confirmed by comparing the approximate solutions with the results of direct numerical integration of the original equation. These studies have shown that the occurrence of nonlinear saturation resulting from the magnetic materials or limitation of power amplifier could lead to complicated dynamic behaviours including large-amplitude motions, quasiperiodic motions and chaotic motions, which cause poor dynamic behaviour of the magnetic bearing and deteriorate the performance of control system.

\section{Two-DOF nonlinear rotor-bearing systems}

The aforementioned studies in Section 3 have greatly enhanced the understanding of the nonlinear dynamics of rotors supported by a two-pole magnetic bearing in the presence of single or double components of nonlinearities. However, from a practical perspective, an advanced model to account for the geometric coordinate coupling appears to provide more appropriate results for nonlinear analysis of more complicated magnetic bearing systems 
such as four-pole pairs or eight-pole pairs of magnetic bearings. The nonlinear modelling of rotor-magnetic bearing systems will be a two-DOF nonlinear system when the geometric co-ordinate coupling is taken into account.

Virgin, Walsh and Knight [1995] studied the effect of coordinate coupling due to the geometry of the pole arrangement on dynamic behaviour. Multiple coexisting solutions and fractal boundaries were obtained. However, they neglected all other important nonlinear components such as nonlinear magnetic force to displacement and force to coil current relationships, hysteresis and saturation of the magnetic material, time delays of the control system, and limitations of the power amplifiers and control current. The effect of cross-coupling and nonlinear force to displacement and force to coil current relationships on the dynamics of a single-mass rotor-magnetic bearing system was numerically investigated by Chinta, Palazzolo and Kascak [1996]. Stable quasi-periodic vibration and period-2 solutions were found. Unfortunately, they employed a very simple four-pole magnetic bearing model and neglected other important nonlinearities such as the saturation of magnetic material, time delays of the control system, and limitations of the power amplifier and control current. Later, Chinta and Palazzolo [1998] derived the equations of motion of a two-DOF mass in a magnetic bearing with geometric coupling between the horizontal and vertical components of rotor motion. The dimensionless equations of motion governing the unbalance response of a rotor are given by

$$
\begin{aligned}
& x^{\prime \prime}=f_{r}-f_{l}+\alpha x\left(f_{t}+f_{b}\right)+E \Omega^{2} \cos (\Omega \tau), \\
& y^{\prime \prime}=f_{t}-f_{b}+\alpha y\left(f_{r}+f_{l}\right)+E \Omega^{2} \sin (\Omega \tau)-G,
\end{aligned}
$$

where $x$ and $y$ are the non-dimensional displacements of the rotor, f's are the magnetic forces, $\alpha$ is the geometric coupling coefficient, $E$ is the non-dimensional eccentricity, $\tau$ 
and $\Omega$ are the dimensionless time and rotor speed, and $G$ is the dimensionless rotor weight.

Stable periodic motion of the forced response was obtained by numerical integration and the approximate method of trigonometric collocation, while the unstable motion was obtained by the collocation method. The local stability of periodic motions and bifurcation behaviour were obtained by Floquet theory. System parameters such as rotor speed, imbalance eccentricity, forcing amplitude, rotor weight and geometric coupling were investigated to find regimes of nonlinear behaviour such as jumps and sub-harmonic motion. It was found that the motion of a rotor in magnetic bearings may undergo cyclicfold bifurcation with an increase of the forcing amplitude and undergo period-doubling bifurcation with an increase of frequency. A cyclic-fold bifurcation causes jump and hysteresis phenomena in the forced response. The response increases continuously until it jumps up to a peak amplitude and then the amplitude decreases continuously. The response for decreasing frequency is not the same as the one for increasing frequency. The region enclosed between jump up and down is the hysteresis region. A small hysteresis region implies a weak non-linearity. Stable period-2 motion takes place after the stable period-1 motion becomes unstable following a period-doubling bifurcation, as shown in Figure 7, where the middle branch after bifurcation of the period-1 motion is unstable.

\section{[Insert Figure 7 here ]}

Ji and Leung [2000] studied the primary resonance response of a rigid rotor-magnetic bearing system by using a perturbation method. It was shown that the steady state response became unstable either via saddle-node bifurcations or via Hopf bifurcations. Ji and Hansen [2001] investigated the nonlinear response of a rotor supported by active magnetic bearings under both primary and internal resonances. The equations of motion 
governing the nonlinear response of the rotor were found to be of the following dimensionless form

$$
\begin{aligned}
& \ddot{x}+2 \mu \dot{x}+\omega^{2} x-\alpha_{1} x^{3}-\alpha_{2} x y^{2}-\alpha_{3} x^{2} \dot{x}-\alpha_{4} \dot{x} y^{2}-\alpha_{5} x \dot{y}^{2}-\alpha_{6} x \dot{x}^{2}-\alpha_{7} x y \dot{y}=2 f \cos \Omega t, \\
& \ddot{y}+2 \mu \dot{y}+\omega^{2} y-\alpha_{1} y^{3}-\alpha_{2} x^{2} y-\alpha_{3} y^{2} \dot{y}-\alpha_{4} x^{2} \dot{y}-\alpha_{5} \dot{x}^{2} y-\alpha_{6} y \dot{y}^{2}-\alpha_{7} x \dot{x} y=2 f \sin \Omega t,(4)
\end{aligned}
$$

where $x$ and $y$ are dimensionless displacement of the rotor, $\alpha$ 's are the coefficients of the nonlinear terms obtained by using a Taylor series expansion of magnetic forces, $\Omega$ is the dimensionless rotating speed of the rotor.

The method of multiple scales was used to obtain four averaged equations that describe the modulation of the amplitudes and phases of vibrations in the horizontal and vertical directions. It was shown that the steady state solutions may lose their stability by either saddle-node bifurcations or Hopf bifurcations. In the regime of multiple coexisting solutions, two stable solutions were found. However, they did not consider saturation and hysteresis of the magnetic material, time delays of the control system, and limitations of the power amplifier and control current. Later, based on the model that is given by equation (4), Ji and Leung [2003] studied the super-harmonic resonance response of the rigid rotor-magnetic bearing system. It was shown that the steady-state superharmonic periodic solutions may lose their stability by either saddle-node or Hopf bifurcations. The system may exhibit many typical characteristics of the behaviour of nonlinear dynamical systems such as multiple coexisting solutions, jump phenomena and sensitive dependence on initial conditions. The effects of the feedback gains and imbalance eccentricity on the nonlinear dynamic behaviour and stability of the system were also studied. Ho, Liu and Yu [2003] studied the effect of a thrust active magnetic bearing on the stability and bifurcation of a rotor-magnetic bearing rotor system using a component mode synthesis method. They focused on the influence of nonlinearities on the stability and bifurcation of periodic motion of the rotor-bearing system subjected to the influences of both journal and 
thrust magnetic bearings and mass eccentricity. The periodic motions and their stability margins were obtained by using the shooting method and path-following technique. It was found that the thrust magnetic bearing and mass eccentricity of the rotor may cause the spillover of system nonlinear dynamics and degradation of the stability and bifurcation of periodic motion, resulting in the whirling motion of the first lateral mode.

By using the asymptotic perturbation method, Zhang and Zhan [2005] investigated nonlinear oscillations and chaotic dynamics of a rotor-magnetic bearing system with eightpole pairs and time-varying stiffness. The stiffness of the magnetic bearings was assumed to be time varying in a periodic form. The resulting dimensionless equations of motion for the rotor-magnetic bearing system with time-varying stiffness in the horizontal and vertical directions were for a two-DOF nonlinear system with quadratic and cubic nonlinearities and parametric excitation. The asymptotic perturbation method was used to obtain the averaged equations for the case of primary parametric resonance and subharmonic resonance. It was found that there existed subharmonic period-3, period-4, period-6, period-7, period-8 motion, quasiperiodic and chaotic oscillations in the rotormagnetic bearing system with time-varying stiffness. The numerical results explored the phenomena of multiple solutions and the soft-spring type and hardening-spring type [Nayfeh and Mook, 1979] in the nonlinear frequency-response curves for the rotormagnetic bearing system. Zhang, Yao and Zhan [2006] then numerically investigated the Shinikov type multi-pulse chaotic dynamics for the rotor-magnetic bearing system, based on the same model developed in Zhang and Zhan [2005]. A new jumping phenomenon was shown to exist in the forced response of the rotor-magnetic bearing system with a time-varying stiffness. 
Amer and Hegazy [2007] studied the nonlinear dynamic behaviour of a rigid rotor supported by active magnetic bearings without including gyroscopic effects in their model. The vibration of the rotor was modelled by coupled second-order nonlinear ordinary differential equations with quadratic and cubic nonlinearities. The steady-state response and stability of the system were studied numerically by applying the method of multiple scales and the frequency response function method. Different shapes of chaotic motion were found to exist by using a phase-plane method. The system parameters were shown to have different effects on the nonlinear response of the rotor. Multiple-valued solutions, jump phenomena, hardening and softening nonlinearity were found to occur in the steady-state response.

Inayat-Hussain [2007] numerically investigated the response of an imbalanced rigid rotor supported by active magnetic bearings. Nonlinearities arising from electromagnetic forcecoil current and force-air gap relationships, and the effects of geometrical cross-coupling were incorporated in the mathematical model of the rotor-bearing system. The response of the rotor was observed to exhibit a rich variety of dynamic behaviour including synchronous, sub-synchronous, quasi-periodic and chaotic vibrations. It was shown that the transition from synchronous rotor response to chaos was via a torus breakdown route [Matsumoto, Chua and Tokunaga, 1987]. With an increase of the rotor imbalance magnitude, the synchronous rotor response was found to undergo a secondary Hopf bifurcation resulting in quasi-periodic vibration.

\section{Nonlinear vibrations of a rotor contacting auxiliary bearings}

Mechanical auxiliary bearings are usually incorporated into magnetic bearing systems to prevent physical interaction between the rotor and stator laminations of magnetic bearings 
and to provide rotor support in the event of bearing failure or during an overload situation. These auxiliary bearings also allow the rotor to temporarily run or safely coast down to a stop for maintenance purposes. The auxiliary bearings are also called "safety touch-down bearings”, "back-up bearings” or "catcher bearings” in the literature. These bearings are usually ball bearings or carbon sleeves located on the stator. The clearance between the inner race of backup bearings and the rotor shaft is usually of the order of half the magnetic bearing clearance.

The loss of the magnetic bearing function during operation may lead to either a transient or persistent contact event between the auxiliary bearings and the magnetically suspended rotor. Subsequent interactions of the rotor and auxiliary bearings may significantly influence the behaviour of the rotor through the generation of very large amplitude vibrations and high instantaneous loads, even if the contact duration is relatively short. In many applications such as space applications, safety is a major concern in the design of a rotor-magnetic bearing system. The rotor-bearing system is required to extend the operation of the rotor on auxiliary bearings by taking the maximum advantage of backup bearings and using backup bearings as true auxiliary bearings to provide support during critical situations in a safe manner. A comprehensive understanding of the dynamics of the rotor drop phenomena is essential to help design better auxiliary bearings.

There are a number of theoretical and experimental studies in the literature concerned with the dynamics of rotors when they are in contact with auxiliary bearings. These studies have been mainly focused on characterizing the transient response to determine the effects of the various bearing parameters, in particular friction and damping coefficients as well as stiffness. 
Gelin et al. [1990] studied the transient dynamic behaviour of rotors landing on auxiliary bearings in an industrial centrifugal compressor. However, the Coulomb friction contact force was ignored in their numerical model. Ishii and Kirk [1991] and Kirk and Ishii [1993] developed a transient response technique for predicting the transient response during the rotor drop for a simple two-mass Jeffcott rotor system after the magnetic bearings became inactive. They showed that an optimum damping could be chosen to prevent destructive backward whirl. Through experimental and theoretical investigation, Schmied and Pradetto [1992] reported on the vibration behaviour of a one-ton compressor rotor being dropped into the auxiliary bearings after the magnetic bearings fail. Fumagalli et al. [1994] classified the touchdown process into four distinct phases of motion-free fall, impact, sliding and rolling — and investigated the influences of such parameters as air gap, friction coefficients and damping on the impact dynamics. Schweitzer, Bleuler and Traxler [1994] presented a comprehensive discussion of issues related to the touch-down dynamics of rotors on auxiliary bearings. Feeny [1994] explored the stability of cylindrical and conical whirls in a perfectly balanced and rigid rotor on rigid retainer bearings. Xie and Flowers [1994] presented a study on the steady state behaviour of a rotor-auxiliary bearing system and reported on its complex dynamic behaviour. Kirk and his co-workers [1994a; 1994b] performed experimental rotor drop tests for balanced and unbalanced conditions, and developed a finite element code for the rotor and bearing system to perform stability analysis and unbalance response. Swanson, Kirk and Wang [1995] discussed experimental data for the initial transient response of a magnetically supported rotor drop on ball and solid auxiliary bearings. Maslen and Barrett [1995] derived whirl conditions of a circularly isotropic rotor and catcher bearing support along with the test results of a commercial compressor rotor with bearings. Tessier [1997] described the development and delevitation tests of a flexible compressor rotor. Foiles and Allaire [1997] presented the nonlinear transient modelling of rotors during rotor drop on auxiliary 
bearings for two types of rotors; generator or turbine rotor and a centrifugal compressor rotor. Chen, Walton and Heshmat [1997] introduced the zero clearance auxiliary bearing which is a specific bearing of planetary elements. The nominal clearance between the machine rotor and auxiliary bearings is zero when the auxiliary bearings are actuated to support the shaft. Experiments showed that the possibility of a backward whirl of a rotor could be reduced due to clearance elimination and damping. Ecker [1997] presented steady state numerical results for a rigid rotor with imbalance on a catcher bearing fixed to the bearing housing.

Wang and Noah [1998] studied the dynamic response of a rotor landed on catcher bearings in a magnetically supported rotor, following loss of power or overload of active magnetic bearings. They constructed an analytical model involving a disk, a shaft and auxiliary bearings on damped flexible supports and developed appropriate equations for the nonlinear dynamic system. The equations included a switch function to indicate contact or non-contact events and determine the existence of contact normal forces and tangential friction forces between the shaft and bearings. The shooting method was used to obtain steady state periodic solutions of the unbalanced rotor for various parameters. It was observed that friction forces could cause both periodic and quasi-periodic large amplitude, full backward whirling. It was found that steady-state, periodic, quasi-periodic and chaotic co-existing solutions may occur for a given range of system parameters. The side forces tend to cause noncircular orbits and the rotor response becomes entangled displaying more complex patterns. Xie, Flowers, Feng and Lawrence [1999] used the harmonic balance method and direct numerical integration to study the steady-state responses of a rotor system supported by auxiliary bearings with a clearance. They discussed the influence of rotor imbalance, clearance, support stiffness and damping on the steady-state behaviour of the rotor motion. Bifurcation diagrams were used as a tool to 
examine the dynamic behaviour of the system as a function of the system parameters. They suggested that auxiliary bearings with low clearance, low support stiffness and high support damping tend to reject the development of multi-frequency and chaotic behaviour, and provide the most favourable rotordynamic behaviour. By summarizing a number of studies concerned with auxiliary bearings, Kirk [1999] reviewed analytical techniques to predict rotor transient response and presented results for the transient response evaluation of a full-size compressor rotor to illustrate some of the important parameters in the design for rotor drop.

Ji and Yu [2000] investigated the transient nonlinear dynamics of a high-speed unbalanced rigid rotor dropping onto rigid sliding bearings. They numerically studied the dynamics of the rotor in different regimes of the touchdown process-free fall, impact, sliding and rolling — and examined the influences of system parameters such as unbalance, air gap, coefficient of friction, and coefficient of restitution on the drop dynamics of the rotor. It was shown that when the unbalance is small, the resulting motion is also small. As the level of unbalance increases, the motion of the rotor becomes larger, so there is potential for damage to the rotor and backup bearings. Zeng [2002; 2003] numerically and experimentally studied the transient response of the rotor motion during the rotor drop when the rotor is supported by backup bearings. It was shown that the nonlinear rotorbackup bearing system would undergo irregular or chaotic motions at some rotating speeds. Under some conditions, the full clearance whirl motion of the rotor in backup bearings may occur, which may lead to damage to the magnetic bearing system. It was shown that optimisation of the parameters characterising the backup device could be used to regulate the nonlinear resonances and hence avoid full clearance whirl motion of the rotor. These parameters include support damping, support stiffness and support device mass. 
Sun, Palazzolo, Provenza and Montague [2004] presented numerical simulations of a rotor drop on catcher bearings in a flywheel energy storage system. They developed a catcher bearing model which includes a Hertzian load-deflection relationship between mechanical contacts, speed-and-preload-dependent bearing stiffness due to centrifugal force, and a Palmgren's drag friction torque. The numerical results showed that friction coefficients, support damping and side loads are critical parameters to satisfy catcher bearing design objectives and prevent backward whirl. Later, Sun [2006a] presented a numerical analysis using detailed catcher bearing and damper models for a rotor drop on catcher bearings, and the resultant thermal growths arising from the subsequent mechanical rub. The catcher bearing model was determined based on the catcher bearing material, geometry, speed and preload, using the nonlinear Hertzian load-deflection formula. The thermal growths of bearing components during the rotor drop were approximated by using a one-dimensional thermal model. Sun [2006b] predicted an estimated fatigue life of a catcher bearing based on the Hertzian contact dynamic loads between bearing balls and races during touchdown. Numerical simulations for an energy-storage flywheel module revealed that a high-speed backward whirl significantly reduces the catcher bearing life and that an optimal damping lowers the catcher bearing temperature and increases the catcher bearing life.

It has been shown that most of studies have been performed from the perspective that the rotor will be shut down if one or more of the magnetic bearings fail. As a result, most of the work to date has concentrated on the transient dynamic behaviour immediately following the failure of a magnetic bearing. However, rotor mass loss, base excited motions and other abnormal operating conditions may lead to transient rotor motion of large amplitude and rotor-auxiliary bearing contacts, even if the magnetic bearing system continues to function. To actively return the rotor to a non-contacting state it is essential to 
determine the manner in which contact events affect the rotor vibration signals used for position control. Toward this aim, Keogh and Cole [2003a] developed an analytical procedure to assess the nature of rotor contact modes under idealized contacts for the case when a magnetic bearing still retains full control capability. Nonlinearities arising from contact and magnetic bearing forces were included in simulation studies involving rigid and flexible rotors to predict rotor response and evaluate rotor synchronous vibration components. It was shown that changes in the synchronous vibration amplitude and phase induced by contact events cause existing controllers to be ineffective in attenuating rotor displacements. The widely used family of synchronous vibration controllers were found to be ineffective when persistent auxiliary bearing contact occurs. The findings were then used as a foundation for the design of new controllers that are able to recover rotor position control under a range of contact cases. As such, Cole and Keogh [2003b] developed a method for robust control of synchronous vibration components that can maintain dynamic stability during interactions between the rotor and auxiliary bearings. The controllers were designed to minimize the severity and duration of contact and ensure that the rotor vibration returns to optimal levels, provided that sufficient control force capacity is available.

\section{Stability and dynamics associated with time delays}

A magnetic bearing system is inherently unstable and thus feedback control must be employed to stabilize the system. Time delays occurring in the feedback control loop are unavoidable especially in digital control systems, even though the control decision process is carried out very quickly. Time delays may have a profound impact on the stability and dynamics of a rotor-magnetic bearing system. There are two sources of time delay in the digital controller loop. First, the A/D and D/A conversions take time. The sample and hold 
devices introduce a delay of half a sampling period. The second source of delay is the controller computation. The amount of computation delay depends upon how the inputs and outputs are synchronized in the controller implementation algorithm. Another source of time delay is the reaction time of the electromagnetic actuators to the control decisions.

Ji [2003a] investigated the effect of time delays occurring in a PID feedback controller on the linear stability of a balanced rotor supported by a two-pole magnetic bearing. It was found that the trivial fixed point of rotor motion might lose its stability through Hopf bifurcations when the time delay crosses certain critical values. Co-dimension two bifurcations of the equilibrium [i.e. bifurcations occurring on varying two control parameters] resulting from non-resonant and resonant Hopf-Hopf interactions were also found to exist in the system. Ji [2003b] also studied the effect of time delays occurring in the proportional-derivative (PD) feedback control loop on the linear stability of a simple magnetic bearing system by analysing the associated characteristic transcendental equation. It was found that a Hopf bifurcation may take place in the autonomous system when time delays pass certain values. The direction and stability of the Hopf bifurcation were determined by applying the normal form method and constructing a center manifold [Guckenhimer and Holmes, 1983; Troger and Steindl, 1991]. It was shown that a bifurcation of co-dimension two may occur through a Hopf and a steady state bifurcation interaction. Ji [2003c] also examined the effect of time delays present in a PD feedback controller on the nonlinear dynamic behaviour of a Jeffcott rotor with an additional magnetic bearing located at the central disc. For the corresponding autonomous system, linear stability analysis was performed by constructing a center manifold. It was found that the trivial solution may lose its stability through either a single or double Hopf bifurcation. For the non-autonomous system, the primary resonance response was studied for its small non-linear motions using the method of averaging. The effects of time delays 
and control gains as well as excitation amplitude on the amplitude of the steady-state response were investigated both theoretically and experimentally. It was shown that the steady state response may exhibit saddle-node and Hopf bifurcations. Increasing the extent of time delays tends to increase the peak amplitude of the response and shift the frequency-response curve to higher frequencies. Large time delays may induce instability of the system.

Based on the model developed by Ji [2003b], Wang and Liu [2005] further investigated the stability of a magnetic bearing system with time delays by analysing the distribution of the roots of the associated characteristic equation. It was found that Hopf bifurcation occurs when the delay passes through a sequence of critical values. The explicit algorithm for determining the direction of the Hopf bifurcations and the stability of bifurcating periodic solutions was derived using the theory of normal form and center manifold [Guckenhimer and Holmes, 1983; Troger and Steindl, 1991]. Later, Wang and Jiang [2006] reported on the multiple stabilities of the magnetic bearing system with time delays. They performed centre manifold reduction and normal form computation for a simple zero singularity and carried out a detailed bifurcation analysis. Some numerical simulations were also presented to illustrate the results found.

Ji and Hansen [2005b] studied the influence of a time delay occurring in a PD feedback controller on the dynamic stability of a rotor suspended by magnetic bearings, by taking geometric coordinate coupling into account. The equations of motion governing the response of the rotor were derived as a set of two-DOF nonlinear differential equations with time delay coupling in the nonlinear terms. It was found that as the time delay increases beyond a critical value, the equilibrium position of the rotor motion becomes unstable and may bifurcate into two qualitatively different kinds of periodic motion. The 
resultant Hopf bifurcation of multiplicity two was found to be associated with two coincident pairs of complex conjugate eigenvalues crossing the imaginary axis. Based on the reduction of the infinite dimensional problem to the flow on a four-dimensional centre manifold, the bifurcating periodic solutions were obtained using a perturbation method. Ji and Hansen [2005c] considered the forced dynamic behaviour of the corresponding nonlinear non-autonomous system in the neighbourhood of the Hopf bifurcation of multiplicity two with the aid of the decomposition theorem and centre manifold theorem. As a result of the interaction between the Hopf bifurcating periodic solutions and the external periodic excitation, primary resonances may occur in the forced response of the system when the forcing frequency is close to the Hopf bifurcating periodic frequency. The method of multiple scales was used to obtain four first-order ordinary differential equations that determine the amplitudes and phases of the phase-locked periodic solutions. The first-order approximations of the periodic solutions were found to be in excellent agreement with those obtained by direct numerical integration of the delay-differential equation. It was also found that the steady state solutions of the nonlinear non-autonomous system may lose their stability via either a pitchfork or Hopf bifurcation. It was shown that the primary resonance response may exhibit symmetric and asymmetric phase-locked periodic motions, quasi-periodic motions, chaotic motions and coexistence of two stable motions. Based on the behaviour of the solutions to the four-dimensional system of ordinary differential equations, Ji, Hansen and Li [2005] investigated the effect of external excitations resulting from unbalance on the dynamic behaviour of the corresponding nonautonomous system following the Hopf bifurcation of the trivial equilibrium of the corresponding autonomous system. It was shown that the interaction between the Hopf bifurcating solutions and the high level excitations may induce a non-resonant or secondary resonance response, depending on the ratio of the frequency of bifurcating periodic motion to the frequency of external excitation. The first-order approximate 
periodic solutions for the non-resonant and super-harmonic resonance response were observed to be in good agreement with those obtained by direct numerical integration of the delay differential equation. It was found that the non-resonant response may be either periodic or quasi-periodic. It was shown that the super-harmonic resonance response may exhibit periodic and quasi-periodic motions as well as a co-existence of two or three stable motions. From a physical point of view, the occurrence of periodic or quasi-periodic stable motions after Hopf bifurcations assures the dynamic stability of the magnetic bearing system. The rotor motion does not diverge but converges to a stable motion after the trivial equilibrium loses its stability. As long as the motion is within the clearance of the auxiliary bearings, the rotor will not contact the backup bearings and the magnetic bearing system could still work well, thereby extending the operating region.

\section{Other issues relevant to nonlinear magnetic bearings}

This section provides an introduction to two merging topics of research in which the nonlinear properties of magnetic bearings are taken into account. These are nonlinear selfsensing magnetic bearings and nonlinear control techniques. Consideration of nonlinearity in the dynamic model for self-sensing magnetic bearings can capture the full potential for nonlinearity to play an essential role in enhancing robustness. The linear feedback controllers designed on the basis of a linearized model cannot be effective across the entire operating region, because the highly nonlinear properties of magnetic bearings may diminish the performance of magnetic bearing systems when the operation departs from the equilibrium point. Nonlinear control techniques are designed to account for the nonlinear properties of magnetic bearing systems and overcome this limitation. 


\subsection{Nonlinear self-sensing magnetic bearings}

The self-sensing (sensorless) magnetic bearing is a special kind of magnetic bearing which uses the same system as both an actuator and a sensor. Self-sensing magnetic bearings use the measurement of voltage and current in electromagnets to estimate the position of a magnetically levitated rotor [Bleuler, 1992; Vischer and Bleuler, 1993; Noh and Maslen, 1997]. By estimating position in this manner, explicit proximity sensors are eliminated. The position information is deduced from the electromagnetic interaction between the stator and rotor. The main advantages of self-sensing magnetic bearings include a reduction in the manufacturing costs, elimination of hardware complexity, simplification of the assembly and maintenance of the magnetic bearing systems, and provision of a more compact design of the rotor-bearing system with higher natural frequencies. Selfsensing magnetic bearings have attracted a lot of attention from the research community, since the problem was first reported in 1990 [Vischer and Bleuler, 1990]. For example, Mizuno and Bleuler [1995] developed a control system for disturbance cancellation of static load and sinusoidal disturbance in self-sensing magnetic bearings by using the geometric approach [Wonham, 1974]. Mizuno, Ishii and Araki [1998] analysed the dynamic characteristics of a hysteresis amplifier for designing new circuits. It was shown that the switching frequency of the amplifier changes linearly with the gap between the electromagnet and the suspended object. Stable suspension was obtained by feeding back the frequency-to-voltage converted switching signal of the hysteresis amplifiers.

One of the obstacles confronting self-sensing technology is the nonlinearity associated with operation of the actuator in its magnetic saturation regime. This problem is especially important in high specific capacity magnetic bearings. Development of a nonlinear model will greatly extend the operating range of self-sensing bearings, as the linear behaviour of 
magnetic bearings can only be achieved approximately locally in a small range of small rotor deflections and small control currents.

Skricka and Markert [2001] explored the effects of cross-axis sensitivity and coordinate coupling on self-sensing by using nonlinear magnetic reluctance models. It was shown that a self-sensing method based on single magnet models might result in large errors in the estimated position. Thus, they suggested that a precise model including nonlinearities of geometric coupling and saturation of the magnetic material need to be developed to predict precisely the behaviour of active magnetic bearings. Later, Skricka and Markert [2002a; 2002b] studied two aspects of the integration of electromagnetic bearings by considering the nonlinearity of magnetic force. The nonlinear component of the magnetic force was compensated by software integrated in the digital controller. The rotor position was identified from the electric state variables directly at the power amplifiers. The realisation of linearized magnetic force was achieved by software using control methods instead of pre-magnetization currents, which reduce unwanted nonlinear effects and the power losses as well as unnecessary energy consumption in imposing the premagnetization currents.

Recently, Maslen, Montie and Iwasaki [2006] developed a linear periodic model of the magnetic bearing system to predict more acceptable levels of robustness than the predictions based on a linear time-invariant model. The essential features of the nonlinearity were retained in their model by linearization along a periodic trajectory. A linear time-invariant model, which is derived from the underlying nonlinear model by linearizing the system at a fixed equilibrium point, was found to be potentially inaccurate for general nonlinear self-sensing magnetic bearings in where nonlinearity may play a crucial role to enhance robustness. 


\subsection{Nonlinear control techniques}

One obstruction to more widespread industrial application of magnetic bearings is the high sensitivity of the control system to parametric uncertainties and bearing nonlinearities [Knospe, 2007; Hung, Albritton and Xia, 2003]. Due to the intractability of the complexity of the actual model, many of the control techniques currently used for active magnetic bearings were generally designed by ignoring the nonlinearity of the magnetic force and the nonlinear effects of the sensors and actuators. The feedback control systems were typically designed using a linearised model of the system, however highly nonlinear properties of the bearing can limit the performance of the overall system. The classical approach for magnetic bearing controller design was to perform a generalized Taylor series linearization about a nominal equilibrium point. Because of the abundant literature available for linear control theory, linear controllers have been applied to magnetic bearing systems extensively. For example, Cho [1993] investigated the application of sliding mode control to stabilise an electromagnetic suspension system for use in vibration isolation platforms and magnetic bearings. Setiawan, Mukherjee and Maslen [2001; 2002] studied synchronous sensor runout and unbalance compensation for magnetic bearing systems. Thibeault and Smith [2002] derived bounds on functions of sensitivity and complementary sensitivity to deduce achievable robustness and performance limits for a single-degree-of-freedom magnetic bearing system in three measurement configurations: measurement of the rotor position for feedback; measurement of the coil currents; and measurement of both position and current. It was shown that the bounds changed with varying magnetic bearing physical dimensions and other parameter values as well as varying the bandwidth of a linear, time-invariant controller. Hu, Lin, Jiang and Allaire 
[2005] developed a systematic control design approach for magnetic bearing systems that are subject to both input and state constraints.

The linear feedback system designs based on linearizing the dynamic equations about the equilibrium point are not valid across the entire operating region, because the controller performance may suffer rapid deterioration when the operation deviates from the equilibrium point. In order to maximize magnetic bearing capabilities where nonlinearity may play a crucial role, the control system needs to properly compensate for the nonlinear dynamics of magnetic bearing systems.

Many nonlinear control techniques have thus been designed to account for the nonlinear magnetic bearing model [for example, De Queiroz, Dawson and Suri, 1998; Li, 1999; Hong and Langari, 2000]. Lei, Palazzolo, Na and Kascak [2000] developed a unique control approach for prescribed large motion control using magnetic bearings in a highspeed compressor wheel. They employed nonlinear fuzzy logic control to the nonlinear magnetic bearing model, which incorporates a nonlinear B-H curve, Ampere's law and a Maxwell stress tensor. Schroder, Green, Grum and Fleming [2001] demonstrated a convenient method for automating a tuning process to produce an optimal design. The magnetic circuit dynamics was included in the modelling of the nonlinear characteristics of the magnetic bearings. It was found that the optimised controllers removed a nonlinear high-to-low-frequency coupling effect. Yeh, Chung and Wu [2001] proposed a sliding control scheme to deal with the nonlinear, uncertain dynamics of magnetic bearing systems. The model characterized both the main electromechanical interaction and the secondary electromagnetic effects such as flux leakage, fringing fluxes and finite core permeance. The controller consisted of two parts: the nominal control part that linearizes 
the nonlinear dynamics, and the robust control part that provides robust performance against the uncertainties.

Hung, Albritton and Xia [2003] designed a nonlinear control system for a magnetic journal bearing using a combination of feedback linearization and backstepping concepts. The derived equations of motion included flux linkage, electromagnetic dynamics, and magneto-mechanical dynamics, as well as a state variable model. Ji and Hansen [2003] developed a linear-plus-nonlinear feedback control strategy to stabilize an unstable Hopf bifurcation in a rotor-magnetic bearing system, for which the linearizied system possesses double zero eigenvalues. The addition of further nonlinear terms was used to modify the coefficients of the nonlinear terms in the reduced normal forms. It was found that feedback control incorporating certain quadratic terms renders the Hopf bifurcation supercritical, thereby extending the operational region of magnetic bearing systems.

\section{Conclusion and future work}

Nonlinear dynamic analysis of magnetic bearing systems is far from complete, though significant efforts have been made in understanding the stability and nonlinear dynamics of magnetic bearing systems. This review has attempted to summarise current research and development in the area of nonlinear dynamics of magnetic bearing systems. It has been shown that published work has highlighted the influences of the nonlinear properties of magnetic bearings and the effects of time delays in the feedback control loops on the dynamic behaviour and stability of rotor-bearing systems incorporating magnetic bearings. The results of existing studies have provided useful information for the design of magnetic bearings and the prediction of their nonlinear dynamic behaviour. The control methods developed have partially compensated certain nonlinear terms associated with magnetic 
forces. This has improved the performance of magnetic bearings including increasing robustness against harmonic disturbances and parameter uncertainties and extending the operational region.

\subsection{Concluding remarks}

Many nonlinear models have been developed in studying the effects of one or more components of the nonlinear properties and the influences of time delays on the stability and nonlinear dynamic behaviour of magnetic bearing systems. Modern dynamical systems theory, perturbation methods, and numerical simulations have been applied to the nonlinear modelling of problems. Experiments have been conducted to validate the theoretical predictions. Current research has provided fundamental concepts of how the time delays, nonlinear magnetic forces, geometric coordinate coupling and saturation constraints can lead to instability and the complex dynamic behaviour of magnetic bearing systems. In particular, many analytical and experimental studies have led to insight on the effects of (1) geometric coupling and the nonlinear magnetic force to displacement and force to coil current relationships on the dynamic behaviour and performance of magnetic bearings; and (2) the nonlinear electromagnetic force incorporating time delays of the control system or saturation of the power amplifier on the dynamic behaviour and performance of magnetic bearings. These studies have been directed towards the exploration of the nonlinear resonant response, local and global bifurcations, and periodically- and chaotically-amplitude modulated responses of rotor-magnetic bearing systems. The studies have also led to a comprehensive understanding of the interaction of the external excitation and bifurcating solutions that immediately follow from Hopf bifurcation of the trivial equilibrium of the corresponding autonomous systems. Research findings provide valuable information for the prediction of bifurcations, instabilities and 
complex responses as well as the online detection of malfunctions of magnetic bearing systems at an early stage of their development before they become catastrophic.

\subsection{Future work}

Although significant efforts have been made to gain a comprehensive understanding of the stability and nonlinear dynamics of magnetic bearing systems, there are many challenging problems that remain unsolved. The following discussion presents some of the key aspects that will drive future research on the nonlinear dynamics and nonlinear modelling of magnetic bearing systems.

Nonlinear modelling of magnetic bearing systems is very challenging because of their highly individual nonlinear nature and complexity. It has been shown that an accurate rotor-magnetic bearing system model with suitable uncertainty descriptions is of critical importance in applying advanced control techniques [Li, Lin, Allaire and Luo, 2006]. An accurate model plays an important role in the dynamic analysis and control design of rotor-magnetic bearing systems due to the complexity involved. Published work has dealt with the most important nonlinearities dominant in magnetic bearing systems. It should be noted that magnetic bearing systems may encounter many less important nonlinearities, such as the nonlinearity of the coil inductance, the nonlinearity of the sensor system, the nonlinearities resulting from the eddy current effect, and the leakage and fringing effect, as discussed in Section 1.2. Useful future research could be directed towards the development of reliable and comprehensive models of complicated magnetic bearings with multiple groupings of nonlinearities, which would enable the treatment of

nonlinearities in large groups including groups containing the less important 
nonlinearities. A preferred system model should include a flexible rotor, magnetic bearings, sensors, amplifier dynamics and digital controllers.

The development of a comprehensive model of magnetic bearings is a formidable task if all of the components of nonlinearities are to be included. Whether or not such a model is possible remains unknown, as some components of nonlinearities are still far from being fully understood and have not yet been accurately identified. Indeed, given the both strong and weak nonlinearities involved in magnetic bearings, such a model, if developed, may be either too hard to be analytically tractable or too complex to be useful. On the other hand, it is not too difficult to envisage improvements to existing models by an inclusion of one or two components of the less important nonlinearities.

The nonlinear magnetic characteristics of high-Tc superconductors and the permanent magnet system are not fully understood either, although Hikihara, Adachi, Moon and Ueda [1999] reported on the dynamic behaviour of a flywheel rotor suspended by a HTSC magnetic bearing and showed a gyroscopic motion under a hysteretic suspension force between high-Tc superconductor and permanent magnets.

In addition to studying the nonlinear dynamics of magnetic bearings using complex models, it is of interest to examine the dynamic behaviour of magnetic bearing systems after single or multiple poles fail. This issue has not been significantly pursued in the literature from the nonlinear dynamics point of view, although existing studies have addressed this issue from the control design point of view by developing fault-tolerant control schemes using linearised magnetic forces and linear system models [Maslen et al., 1999; Chen, 1996; 1999; Sahinkaya, Cole, Keogh and Burrows, 2000; Cole and Burrows, 2001; Na and Palazzolo, 2000; Na, Palazzolo and Provenza, 2002; Na, 2004]. 
System faults can be broadly classified as either internal or external to the magnetic bearing control system [Cole, Keogh, Sahinkaya and Burrows, 2004]. The principal objective of fault-tolerant control is to provide uninterrupted control and high load capacity for continuous operation of the bearing. Failure of a single system component can give rise to destructive rotor dynamic behaviour. In the case of the sudden failure of single or multiple coils, the other coils are required to produce the desired force necessary for suspension. Relatively large increases in current and flux densities would then be required to maintain the stability and similar dynamic properties before and after a failure occurs. However, nonlinearities become strongly significant for large currents and large magnetic forces as well as small air gaps, while linearization about the rotor equilibrium position and nominal perturbation current is valid only for small coil control current variations under constant bias current and small rotor displacements. An understanding of the transient response of a rotor supported by magnetic bearings with one or more failed poles would definitely provide useful information for the detection and control compensation needed to alleviate the effect of pole failures, thereby eliminating the possible occurrence of severe damage to the entire magnetic bearing system. The transient response from normal operation to fault-tolerant control with some coil failures is also of interest. It is anticipated that the transient response of the orbit of the rotor would become elliptic due to asymmetric position stiffness of the failed bearings, which would in turn increase AC power dissipation in the electromagnets and housing vibrations.

Another promising direction for future research appears to be bifurcation control and anticontrol of magnetic bearing systems. Bifurcation control and anti-control deal with modification of system bifurcative characteristics by a designed control input [Chen, Moiola and Wang, 1999; 2000; Chen, Hill and Yu, 2003]. Typical objectives of 
bifurcation control and anti-control include delaying the onset of an inherent bifurcation, stabilizing an unstable solution, introducing a new bifurcation at a preferable parameter value, and optimising the system performance near a bifurcation point. It has been shown that even a simple magnetic bearing system is a rich source of bifurcation phenomena. Saddle-node bifurcations, pitchfork bifurcations and Hopf bifurcations have been found to exist in the nonlinear response of magnetic bearing systems. Unstable bifurcations are unlikely to be of use as they can lead magnetic bearing systems to harmful or even catastrophic situations. In these troublesome cases, unstable bifurcations should be either delayed in their occurrence or eliminated if possible. For example, saddle-node bifurcations may lead to jump and hysteresis phenomena and unstable bifurcations may lead to a divergent dynamic response. Control of such bifurcations not only can significantly improve the performance of magnetic bearing systems, but also can extend the operation regimes. Conventionally, a proportional-differential (PD) or proportionalintegral-differential (PID) feedback controller is used to stabilize the system. Magnetic bearing systems have a significant advantage over other physical systems, as nonlinear feedback strategies can be easily implemented on-line and incorporated into the feedback control system necessary for stable suspension. User-specified controller gains will allow for more flexibility in evaluating the transient and steady state response characteristics by monitoring the instantaneous peak values of all bearing currents, rotor positions, and the lateral velocity and rotational speed of the rotor.

The idea of bifurcation control has been proposed by Ji and Hansen [2003] for stabilizing a sub-critical Hopf bifurcation in a simple magnetic bearing system, so that undesirable unstable behaviour of the system can be prevented. Some possible topics of future research could be directed towards the control of saddle-node bifurcation and pitchfork 
bifurcation as well as Neimark bifurcation which commonly exist in the forced nonlinear response of magnetic bearing systems.

One interesting application of the anti-control of bifurcation would be the creation of a stable Hopf bifurcation in a magnetic bearing system at some preferred parameter values. Creating stable Hopf bifurcations can be viewed as designing limit cycles with specified oscillatory behaviour into a system. A rotor-magnetic bearing system with a fault is generally a complicated nonlinear system, whose behaviour is complex, including quasiperiodic and chaotic vibrations. Monitoring oscillatory behaviour will aid in effective fault diagnosis. The introduction of stable amplitude-modulated motion may serve as a warning signal of an impending failure for magnetic bearings. The controlled system will then exhibit quasi-periodic motions at some preferred values of the system parameters. Anticontrol of bifurcation can also be used to modify the phase-locked response of a magnetic bearing system for intelligent maintenance. The synchronized response may not only be used to extract dynamic features for intelligent maintenance, but would also be used to assess the equipment performance and to detect degradation. For example, measurement of the modified dynamics can be used for on-line monitoring of the response, which will provide useful information for fault diagnosis and maintenance of magnetic bearings. Pole failures, usually caused by a power amplifier failure or coil short circuit, can be catastrophic for magnetic bearings. The occurrence of these failures result in a significant change in the measured dynamics prior to catastrophic failure and measurement of this change can be used as a predictive tool. Due to the response synchronization, only a few sensors will be needed to measure the signals required for the identification of a failure. It is expected that control and anti-control of bifurcation would be valuable techniques for improving and optimising the performance of magnetic bearing systems. 
Another topic of future research could focus on modelling and understanding the nonlinear dynamics of a rotor supported by self-sensing magnetic bearings. A self-sensing magnetic bearing is a special kind of magnetic bearing operating without external position sensors. The position information required by the controller is deduced from the air gap dependent properties of the electromagnets. The main advantage of self-sensing magnetic bearings is the reduction of manufacturing costs. Self-sensing bearings have a number of features that make them interesting. The absence of a position sensor simplifies the construction, the assembly and maintenance of the magnetic bearing system. The rotor position signal necessary for the control system can be generated from the coil currents or the coil impedance which is air gap dependent.

One of the important obstacles confronting self-sensing technology is the nonlinearity associated with the operation of the actuator in its magnetic saturation regime. This problem is especially important for high specific capacity magnetic bearings having high load capacity to weight ratio. Topics of future research relevant to self-sensing magnetic bearings will include the development of a nonlinear theoretical model capable of accurately predicting magnet bearing performance and precisely deriving control signals, as well as a thorough understanding of the effect of nonlinearities on the estimation of the rotor position.

The rapid development of sensing and control technology and further understanding of the nonlinear dynamic behaviour of magnetic bearing systems will definitely lead to the design of more reliable and efficient magnetic bearing systems for many new application fields. 


\section{References}

Aenis, M., Knopf, E., and Nordmann, R. 2002. “Active magnetic bearings for the identification and fault diagnosis in turbomachinery”, Mechatronics, vol.12, pp.10111021.

Allaire, P.E. 1992. Proceedings of the Third International Symposium on Magnetic Bearings, Alexandria, Virginia, USA, Technomic Pub. Co.

Allaire, P.E., and Trumper, D.L. 1998. Proceedings of the Sixth International Symposium on Magnetic Bearings, MIT, Cambridge, MA, USA.

Amer, Y.A., and Hegazy, U.H. 2007. "Resonance behaviour of a rotor-active magnetic bearing with time-varying stiffness”, Chaos, Solitons and Fractals, vol.34(4), pp.13281345.

Bleuler, H. 1992. “A survey of magnetic-levitation and magnetic bearing types”, JSME International Journal Series III-Vibration Control Engineering for Industry, vol.35(3), pp.335-342.

Bleuler, H., and Genta, G. 2006. Proceedings of the Tenth International Symposium on Magnetic Bearings, The Ecole Polytechnique Federale de Lausanne and the Politecnico di Torino, Martigny, Switzerland.

Change, H., and Chung, S.C. 2002. "Integrated design of radial active magnetic bearing systems using genetic algorithms”, Mechatronics, vol.12, pp.19-36.

Chen, G., Moiola, J.L., and Wang, H.O. 1999. "Bifurcations: Control and anti-control”, IEEE Circuits and Systems Newsletter, vol.10(2), pp.1-19.

Chen, G., Moiola, J.L., and Wang, H.O. 2000. "Bifurcation control: theories, methods, and applications”, International Journal of Bifurcation and Chaos, vol.10, pp.511-548. 
Chen, G., Hill, D.J., and Yu, X. 2003. Bifurcation control: Theory and applications, Springer, Berlin.

Chen, H.M. 1996. “A self-healing magnetic bearing”, Proceedings of the Fifth International Symposium on Magnetic Suspension Technology, Kanazawa, Japan, pp.2534.

Chen, H.M. 1999. "Design of a self-healing magnetic bearing”, 53rd Meeting of the Society for Machinery Failure Prevention Technology, Virginia Beach, VA; USA, pp.283292.

Chen, H.M., Walton, J., and Heshmat, H. 1997. “Test of zero clearance auxiliary bearing”, Proceedings of MAG'97 Industrial Conference and Exhibition on Magnetic Bearings, Alexandria, VA, USA, pp.111-119.

Chinta, M., and Palazzolo, A.B. 1998. "Stability and bifurcation of rotor motion in a magnetic bearing”, Journal of Sound and Vibration, vol.214(5), pp.793-803.

China, M., Palazzolo, A.B., and Kascak, A. 1996. "Quasi-periodic vibration of a rotor in a magnetic bearing with geometric coupling”, Proceedings of the Fifth International Symposium on Magnetic Bearings, Kanazawa, Japan, pp.147-152.

Cho, D.D. 1993. "Experimental results on sliding mode control of an electromagnetic suspension”, Mechanical Systems and Signal Processing, vol.7(4), pp.283-292.

Cole, M.O.T., Keogh, P.S., Sahinkaya, M.N., and Burrows, C.R. 2004. "Towards faulttolerant active control of rotor-magnetic bearing systems”, Control Engineering Practice, vol.12, pp.491-501.

Cole, M.O.T., and Keogh, P.S. 2003. "Rotor vibration with auxiliary bearing contact in magnetic bearing systems, Part 2: robust synchronous control for rotor position recovery”, Proceedings of the Institution of Mechanical Engineers Part C-Journal of Mechanical Engineering Science, vol.217, pp.393-409. 
Cole, M.O.T., Keogh, P.S., and Burrows, C.R. 2002. "Control of multifrequency rotor vibration components", Proceedings of the Institution of Mechanical Engineers Part CJournal of Mechanical Engineering Science, vol.216, pp.165-177.

Cole, M.O.T., Keogh, P.S., and Burrows, C.R. 2000. "Fault-tolerant control of rotor/magnetic bearing systems using reconfigurable control with built-in fault detection”, Proceedings of the Institution of Mechanical Engineers Part C-Journal of Mechanical Engineering Science, vol.214(12), pp.1445-1465.

Cole, M.O.T., Keogh, P.S., and Burrows, C.R. 1998. "Vibration control of a flexible/magnetic bearing system subject to direct forcing and base motion disturbances”, Proceedings of the Institution of Mechanical Engineers Part C-Journal of Mechanical Engineering Science, vol.212(7), pp.535-546.

Cveticanin, L. 2005. "Free vibration of a Jeffcott rotor with pure cubic non-linear elastic property of the shaft”, Mechanism and Machine Theory, vol.40(12), pp.1330-1344.

De Queiroz, M.S., Dawson, D.M., and Suri, A. 1998. “Non-linear control of a large-gap 2DOF magnetic bearing system based on a coupled model”, IEE Proceedings Control Theory and Application, vol.145(3), pp.269-276.

Ding, Q., Cooper, J.E., Leung, A.Y.T. 2002. "Hopf bifurcation analysis of a rotor/seal system”, Journal of Sound and Vibration, vol.252(5), pp.817-833.

Ecker, H. 1997. "Steady-state orbits of an AMB-supported rigid rotor contacting the backup bearings”, Proceedings of MAG'97 Industrial Conference and Exhibition on Magnetic Bearings, Alexandria, VA, USA, pp.129-138.

Feeny, B.F. 1994. "Stability of cylindrical and conical motions of a rigid rotor in retainer bearings”, Proceedings of the Fourth International Symposium on Magnetic Bearings, Zurich, Switzerland, pp.219-224. 
Foiles, W.C., and Allaire, P.E. 1997. "Nonlinear transient modelling of active magnetic bearing rotors during rotor drop on auxiliary bearings”, Proceedings of MAG '97 Industrial Conference and Exhibition on Magnetic Bearings, Alexandria, VA, USA, pp.239-244.

Fumagalli, M., Varadi, P., and Schweitzer, G. 1994. "Impact dynamics of high speed rotors in retainer bearings and measurement concepts", Proceedings of the Fourth International Symposium on Magnetic Bearings, Zurich, Switzerland, pp.239-244.

Gelin, A., Pugnet, J.M., and Der Hagopian, J. 1990. "Dynamic behaviour of flexible rotors with active magnetic bearings on safety auxiliary bearings", Proceedings of Third International Conference on Rotordynamics, Lyon, France, pp.503-508.

Guckenheimer, J., and Holmes, P. 1983. Nonlinear oscillations, dynamical systems and bifurcations of vector fields, New York, Springer-verlag.

Halanay, A. 1966. Differential equations, stability, oscillations, time lags, Academic Press, New York.

Hale, J.K. 1971. Oscillations in Nonlinear Systems, McGraw-Hill, London.

Hale, J. 1977. Theory of functional differential equations, Springer, New York.

Hale, J.K., and Verduyn Lunel, S.M. 1983. Introduction to functional differential equations, Springer. New York.

Harsha, S.P. 2006. "Nonlinear dynamic analysis of a high-speed rotor supported by rolling element bearings”, Journal of Sound and Vibration, vol.290(1-2), pp.65-100.

Hayashi, C. 1964. Nonlinear Oscillations in Physical Systems, McGraw-Hill, New York.

Higuchi, T. 1990. Proceedings of the Second International Symposium on Magnetic Bearings, Institute of Industrial Science, University of Tokyo, Tokyo, Japan. 
Hikihara, T., Adachi, H., Moon, F.C., and Ueda, Y. 1999. "Dynamic behaviour of flywheel rotor suspended by hysteretic force of HTSC magnetic bearing”, Journal of Sound and Vibration, vol.228(4), pp.871-887.

Ho, Y.S., Liu, H., and Yu, L. 2003. "Effect of thrust magnetic bearing on stability and bifurcation of a flexible rotor active magnetic bearing system", Transactions of the ASME Journal of Vibration and Acoustics, vol.125, pp.307-316.

Hong, S.K., and Langari, R. 2000. "Robust fuzzy control of a magnetic bearing system subject to harmonic disturbances”, IEEE Transactions on Control Systems Technology, vol.8(2), pp.366-371.

Hu, T., Lin, Z.L., Jiang, W., and Allaire, P.E. 2005. "Constrained control design for magnetic bearing systems”, Transactions of the ASME, Journal of Dynamic Systems, Measurement, and Control, vol.127, pp.601-616.

Hung, J.Y., Albritton, N.G., and Xia, F. 2003. “Nonlinear control of a magnetic bearing system”, Mechatronics, vol.13, pp.621-637.

Inayat-Hussain, J.I. 2007. "Chaos via torus breakdown in the vibration response of a rigid rotor supported by active magnetic bearings”, Chaos, Solitons and Fractals, vol.31(4), pp.912-927.

Ishii, T., and Kirk, R.G. 1991. "Transient response technique applied to active magnetic bearing machinery during rotor drop”, ASME Journal of Rotating Machinery and Vehicle Dynamics, vol.35, pp.191-199.

Ji, J.C. (2003a). Stability and bifurcation in an electromechanical system with time delays, Mechanics Research Communications, vol.30, pp.217-225.

Ji, J.C. (2003b). Stability and Hopf bifurcation of magnetic bearing system with time delay, Journal of Sound and Vibration, vol.259(4), pp.845-856. 
Ji, J.C. (2003c). Dynamics of a Jeffcott rotor-magnetic bearing system with time delays, International Journal of Non-Linear Mechanics, vol.38, pp.1387-1401.

Ji, J.C. (2004). Dynamics of a piecewise linear system subjected to a saturation constraint, Journal of Sound and Vibration, vol.271, pp.905-920.

Ji, J.C., and Hansen, C.H. (2001). Nonlinear oscillations of a rotor in active magnetic bearings, Journal of Sound and Vibration, vol.240(4), pp.599-601.

Ji, J.C., and Hansen, C.H. (2003). Local bifurcation control in a rotor-magnetic bearing system, International Journal of Bifurcation and Chaos, vol.13(4), pp.951-956.

Ji, J.C., and Hansen, C.H. (2004a). Analytical approximation of the primary resonance response of a periodically excited piecewise nonlinear-linear oscillator, Journal of Sound and Vibration, vol.278, pp.327-342.

Ji, J.C., and Hansen, C.H. (2004b). Approximate solutions and chaotic motions of a piecewise nonlinear-linear oscillator, Chaos, Solitons and Fractals, vol.20, pp.1121-1133.

Ji, J.C., and Hansen, C.H. (2005a). On the approximate solution of a piecewise nonlinear oscillator under super-harmonic resonance, Journal of Sound and Vibration, vol.283, pp.467-474.

Ji, J.C., and Hansen, C.H. (2005b). Hopf bifurcation of a magnetic bearing system with time delay, Transactions of the ASME Journal of Vibration and Acoustics, vol.127, pp.362-369.

Ji, J.C., and Hansen, C.H. (2005c). Forced phase-locked response of a nonlinear system with time delay after Hopf bifurcation, Chaos, Solitons and Fractals, vol.25, pp.461-473.

Ji, J.C., Hansen, C.H., and Li, X.Y. (2005d). Effect of external excitation on a nonlinear system with time delay, Nonlinear Dynamics, vol.41, pp.385-402. 
Ji, J.C., and Leung, A.Y.T. (2000). Non-linear behavior of a magnetically supported rotor, Proceedings of the Seventh International Symposium on Magnetic Bearings, ETH Zurich, pp.23-28.

Ji, J.C., and Leung, A.Y.T. (2003). Non-linear oscillations of a rotor-magnetic bearing system under superharmonic resonance conditions, International Journal of Non-Linear Mechanics, vol.38, pp.829-835.

Ji, J.C., and Yu, L. (2000). Drop dynamics of a high-speed unbalanced rotor in active magnetic bearing machinery, Mechanics of Structures and Machine, vol.28(2-3), pp.185200.

Ji, J.C., Yu, L., and Leung, A.Y.T. (2000). Bifurcation behaviour of a rotor supported by active magnetic bearings, Journal of Sound and Vibration, vol.235(1), pp.133-151.

Johnson, M.E., Nascimento, L.P, Kasarda, M., and Fuller, C.R. (2003). The effect of actuator and sensor placement on the active control of rotor unbalance, Transactions of the ASME Journal of Vibration and Acoustics, vol.125, pp.365-373.

Kasarda, M.E.F., Mendoza, H., Kirk, R.G., and Wicks, A. (2004). Reduction of subsynchronouos vibrations in a single-disk rotor using an active magnetic damper, Mechanics Research Communications, vol.31, pp.689-695.

Kasarda, M.E.F. (2000). An overview of active magnetic bearing technology and applications, The Shock and Vibration Digest, vol.32(2), pp.91-99.

Keogh, P.S., and Cole, M.O.T. (2003). Rotor vibration with auxiliary bearing contact in magnetic bearing systems, Part 1: synchronous dynamics, Proceedings of the Institution of Mechanical Engineers Part C-Journal of Mechanical Engineering Science, vol.217, pp.377-392.

Keogh, P.S., Cole, M.O.T., and Burrows, C.R. (2002). Multi-state transient rotor vibration control using sampled harmonics, Transactions of the ASME Journal of Vibration and Acoustics, vol.124, pp.186-197. 
Kim, H.Y., and Lee, C.W. (2006). Design and control of active magnetic system with Lorentz force-type axial actuator, Mechatronics, vol.16, pp.13-20.

Kim, S.J., and Lee, C.W. (1999). On-line identification of current and position stiffness by LMS algorithm in active magnetic bearing system equipped with force transducers, Mechanical Systems and Signal Processing, vol.13(5), pp.681-690.

Kim, Y.B., and Choi, S.K. (1997). A multiple harmonic balance method for the internal resonant vibration of a non-linear Jeffcott rotor, Journal of sound and Vibration, vol.208(5), pp.745-761.

Kirk, R.G., Swanson, E.E., Kavarana, F.H., and Wang, X. (1994a). Rotor drop test stand for AMB rotating machinery. Part I: description of test stand and initial results, Proceedings of the Fourth International Symposium on Magnetic Bearings, Zurich, Switzerland, pp.207-212.

Kirk, R.G., Ramesh, K., Swanson, E.E., Kavarana, F.H., and Wang, X. (1994b). Rotor drop test stand for AMB rotating machinery. Part II: steady state analysis and comparison to experimental results, Proceedings of the Fourth International Symposium on Magnetic Bearings, Zurich, Switzerland, pp.213-218.

Kirk, R.G. (1999). Evaluation of AMB turbomachinery auxiliary bearings, Transactions of the ASME Journal of Vibration and Acoustics, vol.121(2), pp.156-161.

Kirk, R.G., and Ishii, T. (1993). Transient rotor drop analysis of rotors following magnetic bearing power outage, Proceedings of MAG'93 Magnetic Bearings, Magnetic Drives, and Dry Gas Seals Conference \& Exhibition, Alexandria, VA, USA, pp.53-61.

Knospe, C.R. (2007). Active magnetic bearings for machining applications, Control Engineering Practice, vol.15(3), pp.307-313.

Knospe, C.R., and Tamer, S.M. (1997). Experiments in robust control of rotor unbalance response using magnetic bearings, Mechatronics, vol.7(3), pp.217-229. 
Knospe, C.R., Hope, R.W., Fedigan, S.J., and Williams, R.D. (1995). Experiments in the control of unbalance response using magnetic bearings, Mechatronics, vol.5(4), pp.385400.

Laier, D., and Markert, R. (1995). Simulation of nonlinear effects on magnetically suspended rotors, Proceedings of the First Conference on Engineering Computation and Computer Simulation ECCS-1, Changsha, China, vol.I, pp.473-482.

Lee, C.W., and Jeong, H.S. (1996). Dynamic modelling and optimal control of coneshaped active magnetic bearing systems, Control Engineering Practice, vol.4(10), pp.1393-1403.

Lei, S.L., Palazzolo, A., Na, U.J., and Kascak, A. (2000). Non-linear fuzzy logic control for forced large motions of spinning shafts, Journal of Sound and Vibrations, vol.235(3), pp.435-449.

Li, G.X., Lin, Z.L., Allaire, P.E., and Luo, J.H. (2006). Modelling of a high speed rotor test rig with active magnetic bearings, Transactions of the ASME Journal of Vibration and Acoustics, vol.128, pp.269-281.

Li, L. (1999). Linearizing magnetic bearing actuators by constant current sum, constant voltage sum and constant flux sum, IEEE Transactions on Magnetics, vol.35(1), pp.528535.

Loesch, F. (2001). Two remarks on the modeling of active magnetic bearing system, Proceedings of the Sixth International Symposium on Magnetic Suspension Technology, Turin, Italy, pp.422-427.

Lyndon, S.S., and Trupmer, D.L. (2004). Proceedings of the Ninth International Symposium on Magnetic Bearings, The University of Kentucky Bearings and Seals Laboratory, Lexington, Kentucky, USA. 
Maccari, A. (1998). Approximate solution of a class of nonlinear oscillators in resonance with a periodic excitation, Nonlinear Dynamics, vol.15, pp.329-343.

Mani, G., Quinn, D.D., and Kasarda, M. (2006). Active health monitoring in a rotating cracked shaft using active magnetic bearings as force actuators, Journal of Sound and Vibration, vol.294, pp.454-465.

Maslen, E.H., and Barrett, L.E. (1995). Feasible whirl of rotors in auxiliary bearings, Proceedings of MAG'95 Magnetic Bearings, Magnetic Drives and Dry Gas Seals Conference \& Exhibition, Alexandria, VA, USA, pp.217-226.

Maslen, E.H., Montie, D.T., and Iwasaki, T. (2006). Robustness limitations in self-sensing magnetic bearings, Transactions of the ASME, Journal of Dynamic Systems, Measurement, and Control, vol.128, pp.197-203.

Maslen, E.H., Sortore, C.K., Gillies, G.T., Williams, R.D., Fedigan, S.J., and Aimone, R.J. (1999). Fault tolerant magnetic bearings, ASME Journal of Engineering for Gas Turbines and Power, vol.121, pp.504-508.

Matsumoto, T., Chua, L.O., and Tokunaga, R. (1987). Chaos via torus breakdown, IEEE Transactions on Circuits and Systems, Vol.34(3), pp.240-253, 1987.

Matsumura, F., Okada, F.Y., Fujita, M., and Namerikawa, T. (1996). Proceedings of the Fifth International Symposium on Magnetic Bearings, Kanazawa, Japan.

Matsushita, O., Imashima, T., and Okubo, H. (2002). Aseismic vibration control of flexible rotors using active magnetic bearings, Transactions of the ASME Journal of Vibration and Acoustics, vol.124, pp.49-57.

Mizuno, T., Ishii, T., and Araki, K. (1998). Self-sensing magnetic suspension using hysteresis amplifiers, Control Engineering Practice, vol.6, pp.1133-1140.

Mizuno, T., and Bleuler, H. (1995). Self-sensing magnetic bearing control system design using the geometric approach, Control Engineering Practice, vol.13(7), pp.925-932. 
Mohamed, A.M., and Emad, F.P. (1993). Non-linear oscillations in magnetic bearing systems, IEEE Transactions on Automatic Control, vol.38(8), pp.1242-1245.

Na, U.J. (2004). Fault tolerance of homopolar magnetic bearings, Journal of Sound and Vibration, vol.272, pp.495-511.

Na, U.J., Palazzolo, A.B., and Provenza, A. (2002). Test and theory correlation study for a flexible rotor on fault-tolerant magnetic bearings, Transactions of the ASME Journal of Vibration and Acoustics, vol.124, pp.359-366.

Na, U.J., and Palazzolo, A. (2001). The fault-tolerant control of magnetic bearings with reduced controller outputs, Transactions of the ASME, Journal of Dynamic Systems, Measurement, and Control, vol.123, pp.219-224.

Na, U. J., and Palazzolo, A. (2000). Optimized realization of fault-tolerant heteropolar magnetic bearings, Transactions of the ASME Journal of Vibration and Acoustics, vol.122, pp.209-221.

Nayfeh, A.H., and Mook, D.T. (1979). Nonlinear Oscillations, Wiley-Interscience, New York.

Noh, M.D., and Maslen, E.H. (1997). Self-sensing magnetic bearings using parametric estimation, IEEE Transactions on Instrumentation and Measurement, vol.46(1), pp.45-50.

Okada, Y., and Nonami, K. (2002). Proceedings of the Eighth International Symposium on Magnetic Bearings, Mito, Japan.

Parker, T.S., and Chua, L.O. (1989). Practical numerical algorithms for chaotic systems, Berlin, New York: Springer Verlag, 1989.

Pavlovskaia, E.E., Karpenko, E.V., Wiercigroch, M. (2004). Nonlinear dynamic interactions of a Jeffcott rotor with preloaded snubber ring, Journal of Sound and Vibration, vol.276, pp.361-379. 
Peel, D.J., Bringham, C.M., and Howe, D. (2002). Simplified characteristics of active magnetic bearings, Proceedings of the Institution of Mechanical Engineers Part C-Journal of Mechanical Engineering Science, vol.216(5), pp.623-628(6).

Quinn, D., Mani, G., Kasarda, M., Bash, T., Inman, D., Kirk, R.G. (2005). Damage detection of a rotating cracked shaft using an active magnetic bearing as a force actuator: Analysis and experimental verification, IEEE/ASME Transactions on Mechatronics, vol.10(6), pp.640-647.

Rudiger, S. (1994). Practical bifurcation and stability analysis (2nd Edition), Springer, New York.

Sahinkaya, M.N., Cole, M.O.T., and Burrows, C.R. (2001). Fault detection and tolerance in synchronous vibration control of rotor-magnetic bearing systems, Proceedings of the Institution of Mechanical Engineers Part C-Journal of Mechanical Engineering Science, vol.215(12), pp.1401-1416.

Schroder, P., Green, B., Grum, N., and Fleming, P.J. (2001). On-line evolution of robust control systems: an industrial active magnetic bearing application, Control Engineering Practice, vol.9, pp.37-49.

Schweitzer, G. (1989). Magnetic bearings-Proceedings of the First International Symposium, ETH Zurich, Switzerland. Springer-verlag, Berlin, Heidelberg.

Schweitzer, G., Bleuler, H., and Traxler, A. (1994). Active Magnetic Bearings, basics, properties and applications of active magnetic bearings, vdf, ETH-Zurich, Zurich, Switzerland.

Schweitzer, G., Siegwart, R., and Herzog, R. (1994). Proceedings of The Fourth International Symposium on Magnetic Bearings, verlag der Fachvereine (vdf), ETHZurich (Swiss Federal Institute of Technology), Zurich, Switzerland. 
Schweitzer, G., Siegwart, R., Lösch, F., and Berksun, R. (2000). Proceedings of The Seventh International Symposium on Magnetic Bearings, ETH-Zurich (Swiss Federal Institute of Technology), Zurich, Switzerland.

Schmied, J., and Pradetto, J.C. (1992). Behaviour of a one ton rotor being dropped into auxiliary bearings, Proceedings of the Third International Symposium on Magnetic Bearings, Alexandria, VA, pp.145-156.

Setiawan, J.D., Mukherjee, R., and Maslen, E.H. (2002). Synchronous sensor runout and unbalance compensation in active magnetic bearings using bias current excitation, Transactions of the ASME, Journal of Dynamic Systems, Measurement, and Control, vol.124, pp.14-24.

Setiawan, J.D., Mukherjee, R., and Maslen, E.H. (2001). Adaptive compensation of sensor runout for magnetic bearings with uncertain parameters: Theory and Experiments, Transactions of the ASME, Journal of Dynamic Systems, Measurement, and Control, vol.123, pp.211-218.

Shi, J., Zmood, R., and Qin, L. (2004). Synchronous disturbance attenuation in magnetic bearing systems using adaptive compensating signals, Control Engineering Practice, vol.12, pp.283-290.

Skricka, N., and Markert, R. (2001). Influence of cross-axis sensitivity and coordinate coupling on self-sensing, Proceedings of the Sixth International Symposium on Magnetic Suspension Technology, Turin, Italy, pp.179-184.

Skricka, N., and Markert, R. (2002a). Improvements in the integration of active magnetic bearings, Control Engineering Practice, vol.10, pp.917-922.

Skricka, N., and Markert, R. (2002b). Improvements of the integration of active magnetic bearings, Mechatronics, vol.12, pp.1059-1068. 
Springer, H., Schlager, G., and Platter, T. (1998). A non-linear simulation model for active magnetic bearing actuators, Proceedings of the Sixth International Symposium on Magnetic Bearings, MIT USA, pp.189-203.

Steinschaden, N., and Springer, H. (1999a). Some nonlinear effects of magnetic bearings, Proceedings of the 1999 ASME Design Engineering Technological Conference, Las Vagas, Nevada, ASME Conf. Paper No. DETC99/VIB-8063.

Steinschaden, N., and Springer, H. (1999b). Nonlinear stability analysis of active magnetic bearings, Proceedings of the Fifth International Symposium on Magnetic Suspension Technology, Santa Barbara, California, pp.411-427.

Sun, G., Palazzolo, A.B., Provenza, A., and Montague, G. (2004). Detailed ball bearing model for magnetic suspension auxiliary service, Journal of Sound and Vibration, vol.269, pp.933-963.

Sun, G. (2006a). Rotor drop and following thermal growth simulations using detailed auxiliary bearing and damper models, Journal of Sound and Vibration, vol.289, pp.334359.

Sun G.Y. (2006b). Auxiliary bearing life prediction using Hertzian contact bearing model, Transactions of the ASME Journal of Vibration and Acoustics, vol.128, pp.203-209.

Swanson, E.E., Kirk, R.G., and Wang, J. (1995). AMB rotor drop initial transient on ball and solid bearings, Proceedings of MAG'95 Magnetic Bearings, Magnetic Drive and Dry Gas Seals Conference \& Exhibition, Alexandria, VA, USA, pp.207-216.

Tessier, L.P. (1997). The development of an auxiliary bearing landing system for a flexible AMB-supported hydrogen process compressor rotor, Proceedings of MAG'97 Industrial Conference and Exhibition on Magnetic Bearings, Alexandria, VA, USA, pp.120-128. 
Thibeault, N.M., and Smith, R.S. (2002). Magnetic bearing measurement configurations and associated robustness and performance limitations, Transactions of the ASME, Journal of Dynamic Systems, Measurement, and Control, vol.124, pp.589-698.

Tonoli, A., and Bornemann, H.J. (1998). Analysis of losses due to rotor vibrations in a high-Tc superconducting flywheel system, Journal of Sound and Vibration, vol.212(4), pp.649-662.

Troger, H., and Steindl, A. (1991). Nonlinear stability and bifurcation theory, Wien, New York: Springer verlag.

Virgin, L., Walsh, T.F., and Knight, J.D. (1995). Non-linear behavior of a magnetic bearing system, ASME Journal of Engineering for Gas Turbines and Power, vol.115(3), pp.582-588.

Vischer, D., and Bleuler, H. (1990). A new approach to sensorless and voltage controlled AMBs based on network theory concepts, Proceedings of the Second International Symposium on Magnetic Bearings, pp.301-306, Tokyo, Japan Tokyo.

Vischer, D., and Bleuler, H. (1993). Self-sensing active magnetic-levitation, IEEE Transactions on Magnetics, vol.29(2), pp.1276-1281.

Wang, H.B., and Liu, J.Q. (2005). Stability and bifurcation analysis in a magnetic bearing system with time delays, Chaos, Solitons and Fractals, vol.26, pp.813-825.

Wang, H.B., and Jiang W.H. (2006). Multiple stabilities analysis in a magnetic bearing system with time delays, Chaos, Solitons and Fractals, vol.27, pp.789-799.

Wang, X., and Noah, S. (1998). Nonlinear dynamics of a magnetically suspended rotor on safety auxiliary bearings, Transactions of the ASME Journal of Vibration and Acoustics, vol.120(2), 596-606.

Wiggins, S. (1990). Introduction to applied nonlinear dynamical systems and chaos, New York, Springer-verlag. 
Wolf, A., Swift, J.B., Swinney, H.L., and Vastano, J.A. (1985). Determining Lyapunov exponents from a time series, Physica D, vol.16, pp.285-317.

Wonham, W.M. (1974). Linear multivariable control-A geometric approach, Berlin, Springer-verlag.

Xie, H., and Flowers, G.T. (1994). Steady-state dynamic behaviour of an auxiliary bearing supported rotor system, ASME Winter Annual Meetings, Chicago, Illinois, pp.13-18.

Xie, H., Flowers, G.T., Feng, L., and Lawrence, C. (1999). Steady-state dynamic behaviour of a flexible rotor with auxiliary support from a clearance bearing, Transactions of the ASME Journal of Vibration and Acoustics, vol.121(1), pp.78-83.

Yeh, T.J., Chung, Y.J., and Wu, W.C. (2001). Sliding control of magnetic bearing systems, Transactions of the ASME Journal of Dynamic Systems, Measurement, and Control, vol.123, pp.353-362.

Zeng, S. (2002). Motion of AMB rotor in backup bearings, Transactions of the ASME Journal of Vibration and Acoustics, vol.124, pp.460-464.

Zeng, S. (2003). Modelling and experimental study of the transient response of an active bearing rotor during rotor drop on back-up bearings, Proceedings of the Institution of Mechanical Engineers Part C-Journal of Mechanical Engineering Science, vol. 217(6), pp.505-517.

Zhang, W., and Zhan, X.P. (2005). Periodic and chaotic motions of a rotor-active magnetic bearing with quadratic and cubic terms and time-varying stiffness, Nonlinear Dynamics, vol.41, pp.331-359.

Zhang, W., Yao, M.H., and Zhan, X.P. (2006). Multi-pulse chaotic motions of a rotoractive magnetic bearing system with time-varying stiffness, Chaos, Solitons and Fractals, vol.27, pp.175-186. 


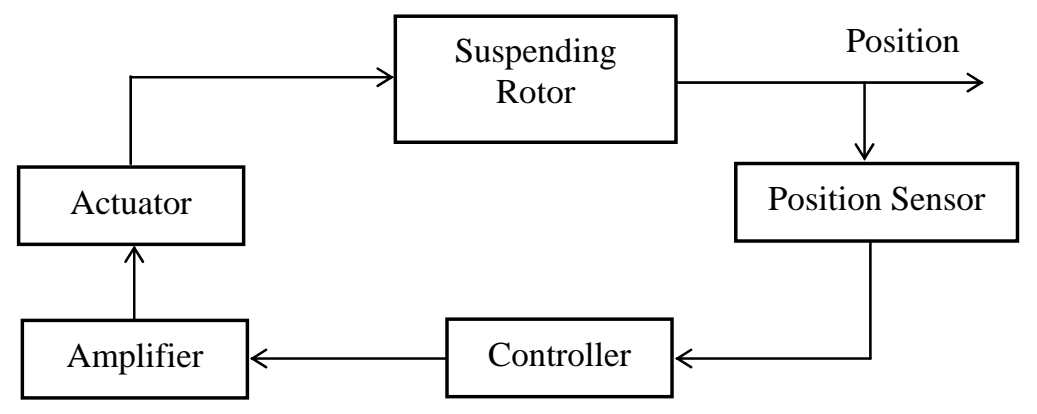

Figure 1 Block diagram of a simple magnetic bearing system. 


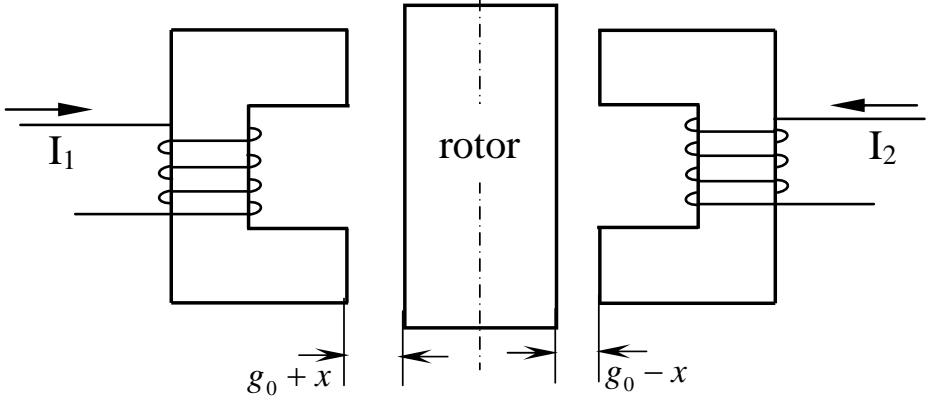

Figure 2 Schematic of a two-electromagnet magnetic bearing. 


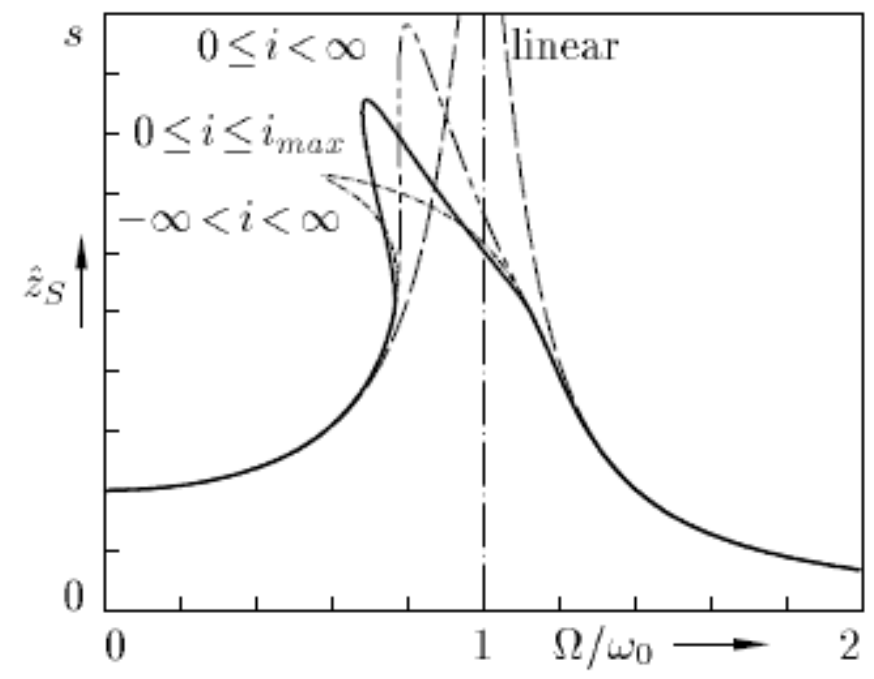

Figure 3: Influence of the limitation of currents on amplitude curves. From Laier and Markert [1995]. 


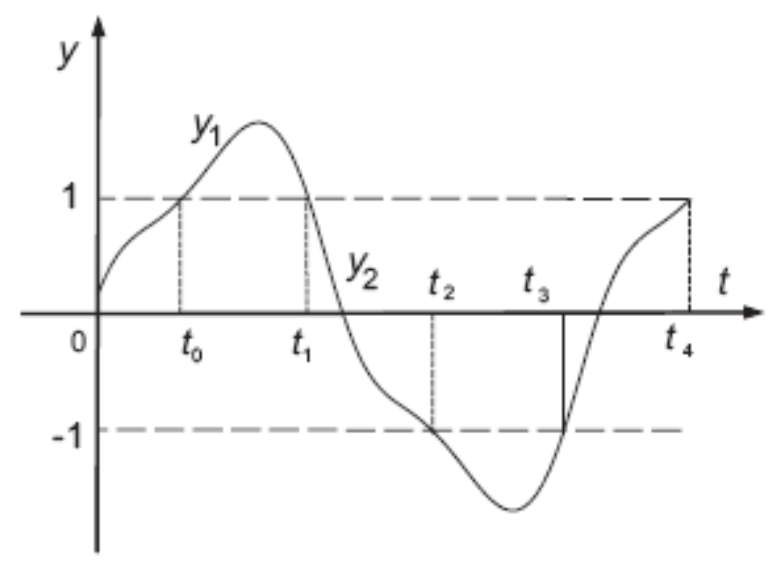

Figure 4 The double-entering saturation region per cycle symmetric period-one solution. From Ji [2004]. 

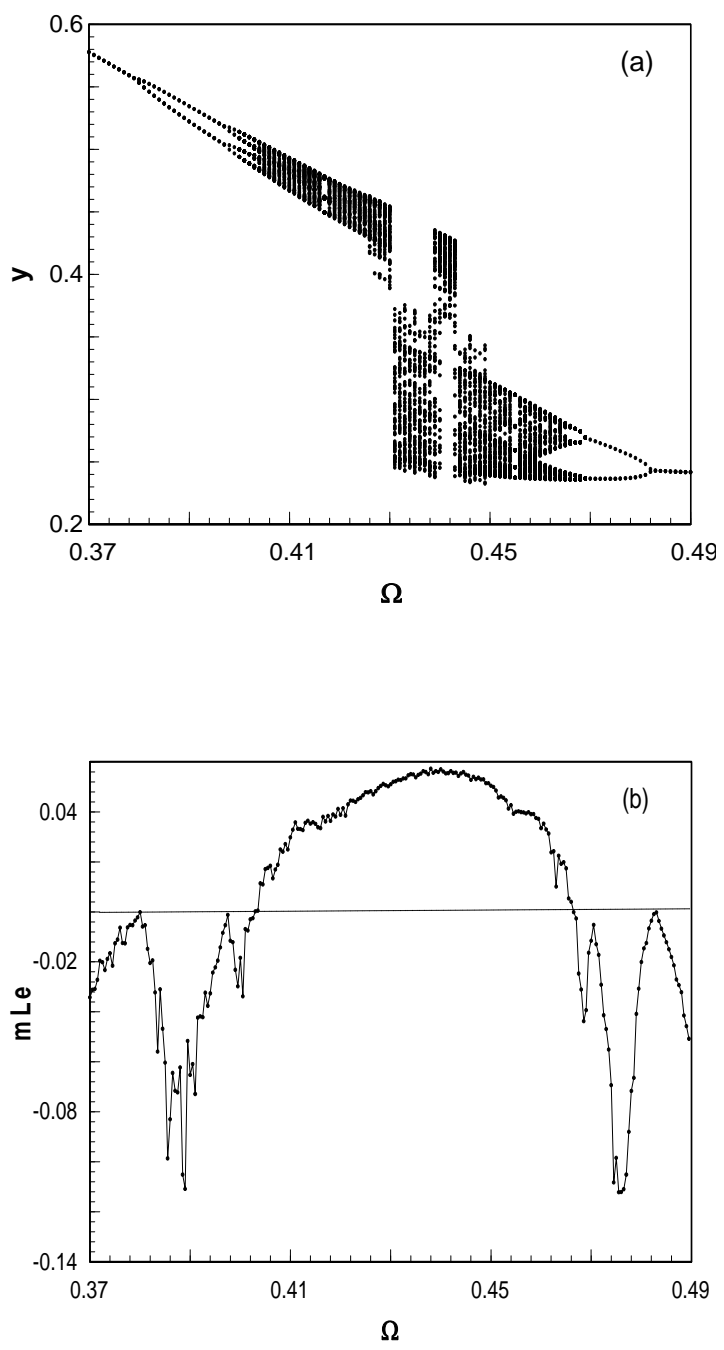

Figure 5. Bifurcation diagram and the maximum Lyapunov exponent (mLe) with an increase of the forcing frequency for $f=0.66$ in the region $\Omega \in[0.37,0.49]$; (a) bifurcation diagram, (b) the maximum Lyapunov exponent (mLe). From Ji and Hansen [2004b]. 

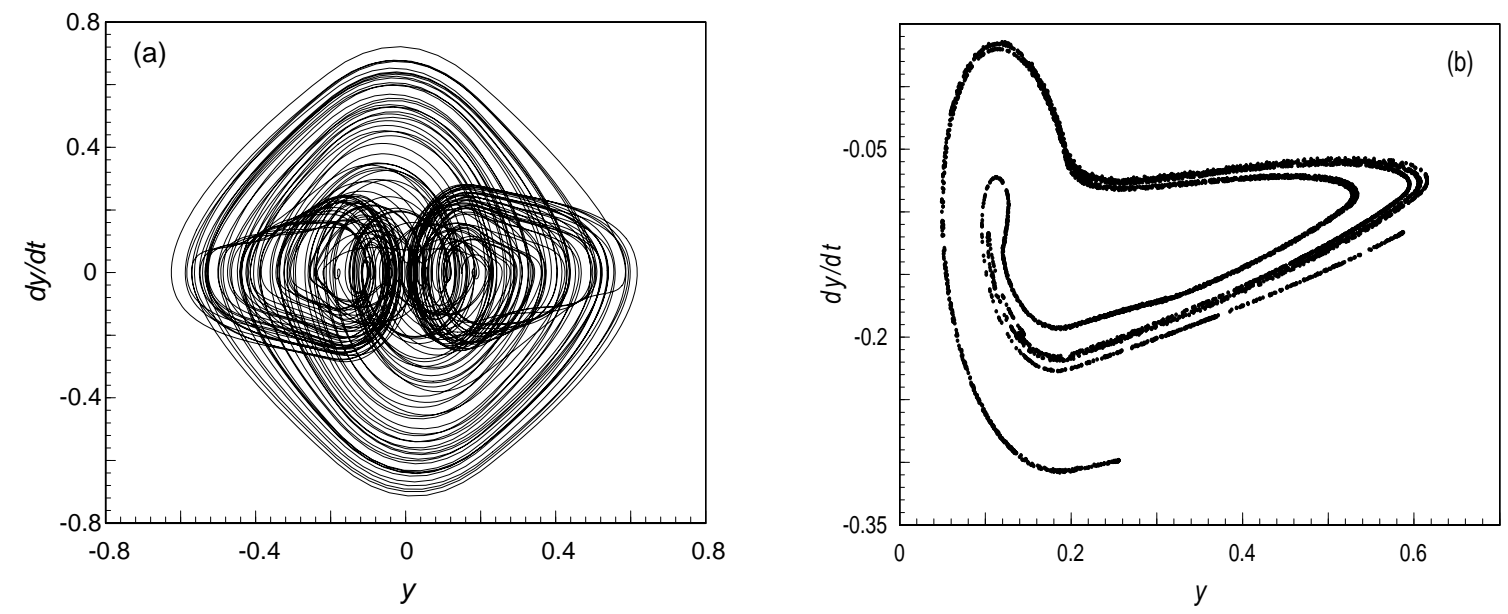

Figure 6. Chaotic response of the system for $f=0.68$ : (a) phase portrait, (b) Poincare map. From Ji and Hansen [2004b]. 


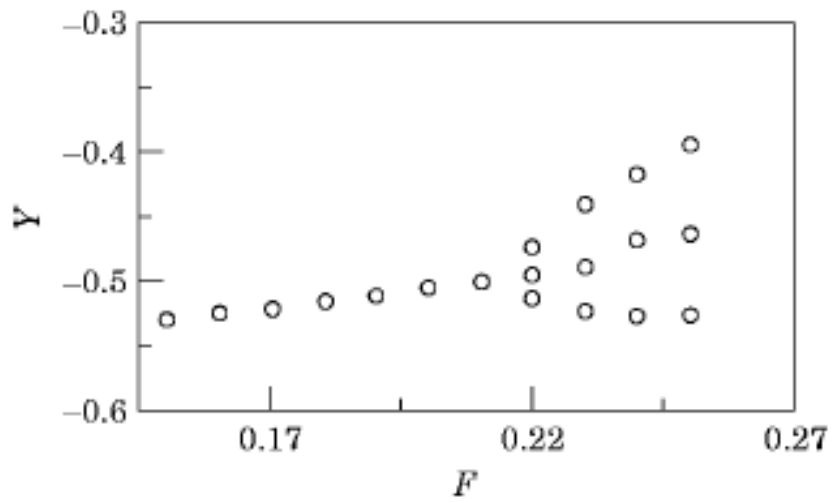

Figure 7. Period-doubling bifurcation: Collocation method; $P=1.1, D=0.03, G=0.03$, $\Omega=1, \alpha=0.16$. From Chinta and Palazzolo [1998]. 